\title{
Implementing and testing the AM05 spin density functional
}

Ann E. Mattsson and Rickard Armiento

\section{Linköping University Post Print}

N.B.: When citing this work, cite the original article.

Original Publication:

Ann E. Mattsson and Rickard Armiento, Implementing and testing the AM05 spin density functional, 2009, Physical Review B. Condensed Matter and Materials Physics, (79), 15, 155101.

http://dx.doi.org/10.1103/PhysRevB.79.155101

Copyright: American Physical Society

http://www.aps.org/

Postprint available at: Linköping University Electronic Press

http://urn.kb.se/resolve?urn=urn:nbn:se:liu:diva-86292 


\title{
Implementing and testing the AM05 spin density functional
}

\author{
Ann E. Mattsson ${ }^{1} *$ and Rickard Armiento ${ }^{2}$ \\ ${ }^{1}$ Multiscale Dynamic Materials Modeling MS 1322, Sandia National Laboratories, Albuquerque, New Mexico 87185-1322, USA \\ ${ }^{2}$ Physics Institute, University of Bayreuth, D-95440 Bayreuth, Germany
}

(Received 4 December 2008; revised manuscript received 18 February 2009; published 2 April 2009)

\begin{abstract}
We show that the spin density generalization of the AM05 density functional [R. Armiento and A. E. Mattsson, Phys. Rev. B 72, 085108 (2005)] predicts the correct ground spin state for iron, a system known to be heavily dependent on proper spin treatment. Using the fundamental assumptions in the subsystem functional scheme, we resolve an ambiguity in how to treat the separate spin densities in AM05 but also show that the other less preferred treatments give no significantly different numerical outcome of the iron body-centeredcubic and face-centered-cubic test cases. Details and formulas are given to aid in the implementation of functionals in general, and the spin-resolved AM05 exchange-correlation potentials in particular, into different types of computer codes.
\end{abstract}

DOI: 10.1103/PhysRevB.79.155101

PACS number(s): 71.15.Mb, 31.15.ej, 71.45.Gm, 75.50.Bb

\section{INTRODUCTION}

Due to its ability to treat a wide range of systems fairly accurately at relatively low computational cost, densityfunctional theory (DFT) (Refs. 1 and 2) and its spin formulation ${ }^{3,4}$ has become the foundation of most large scale quantum-mechanical simulations. The key to accuracy in DFT based simulations is the approximation made for the exchange-correlation density functional.

A number of recent studies have confirmed the excellent performance of the AM05 functional ${ }^{5}$ for nonmagnetic solids. ${ }^{6}$ Furthermore, Ropo et $a .^{7}$ recently investigated a limited set of properties also of magnetic solids using a spinresolved version of AM05 (the xscst version discussed in Sec. II). Recent work by Haas et al. ${ }^{8}$ confirms the findings in Refs. 6 and 7. The present paper presents the necessary details in the derivation of spin-resolved AM05 and discusses its implementation into various types of computer codes. In addition, we test the spin-resolved functional for proper prediction of body-centered-cubic (bcc) and face-centered-cubic (fcc) phases of iron. This is a classical test case, for which the local spin density approximation (LSDA) fails to give the correct ground spin state while, e.g., the Perdew, Burke, and Ernzerhof (PBE) functional ${ }^{9}$ reproduces it correctly.

In Sec. II we give an overview of the AM05 density functional from the subsystem functional viewpoint and we derive several forms of AM05 that naturally extends its use to systems where a spin-resolved electron density is needed for proper treatment. The best form to use is identified based on fundamental subsystem functional principles. Section III discusses the general implementation of spin-resolved functionals in different types of codes currently in use, and we give specific formulas for the spin-resolved AM05. Section IV gives the results of benchmark tests on iron with the positive result that spin-resolved AM05 correctly identifies the ground spin state. We summarize and discuss our findings in Sec. V.

\section{AM05 AND SPIN}

The AM05 functional $^{5}$ consists of a subsystem functiona ${ }^{10,11}$ for interior regions, a subsystem functional for edge regions, and an interpolation index that in each point in a system determines the ratio of edge and interior functionals to use. A subsystem functional is designed to properly treat a particular type of physical situation. AM05 thus combines a treatment based on the uniform electron gas, describing a situation where electrons are free to move in all directions, appropriate for interior regions, with a treatment of a very different edgelike situation, where electrons are confined by a surface, restricting their motion in one direction. AM05 is using only the density, $n(\mathbf{r})$, and the magnitude of its gradient, $|\nabla n(\mathbf{r})|$, to determine the contribution to the total exchange-correlation energy in each point, $\mathbf{r}$, of the system, just as common generalized gradient approximation (GGA) functionals do.

As the interior region functional, the local-density approximation (LDA) (Ref. 2) with the Perdew-Wang (PW) (Ref. 12) parametrization of the Ceperly-Alder data ${ }^{13}$ for correlation is used, $\epsilon_{c}^{\mathrm{LDA}}(n)=\epsilon_{c}^{\mathrm{PW}}(n)$ :

$$
\epsilon_{x c}^{\text {interior }}[n]=\epsilon_{x}^{\mathrm{LDA}}(n)+\epsilon_{c}^{\mathrm{LDA}}(n) .
$$

As edge region functional, an exchange derived from the Airy Gas system ${ }^{5,14}$ [the local Airy approximation (LAA)] is used together with a scaled PW LDA correlation:

$$
\epsilon_{x c}^{\text {edge }}[n]=\epsilon_{x}^{\mathrm{LAA}}(n, s)+\gamma \epsilon_{c}^{\mathrm{LDA}}(n) \quad \gamma=0.8098,
$$

where

$$
s=s(n,|\nabla n|)=\frac{|\nabla n|}{2 k_{F}(n) n}, \quad k_{F}(n)=\left(3 \pi^{2} n\right)^{1 / 3}
$$

is a dimensionless scaled gradient also used by many other GGA-type functionals, and we have suppressed the $\mathbf{r}$ dependence of the density; $n=n(\mathbf{r})$.

The interpolation index,

$$
X(s)=\frac{1}{1+\alpha s^{2}}, \quad \alpha=2.804,
$$

is only dependent on $s$, and determines the ration of edge vs interior functional to use in each point: $X(0)=1, X(s) \rightarrow 0$ when $s \rightarrow \infty$, and 


$$
\epsilon_{x c}^{\mathrm{AM} 05}(n, s)=\epsilon_{x c}^{\text {interior }}(n) X(s)+\epsilon_{x c}^{\text {edge }}(n, s)[1-X(s)] .
$$

Surface physics is included in AM05 by the use of the Airy gas system, and by the constants $\alpha$ and $\gamma$ determined from a fit to the jellium surface ${ }^{15}$ RPA+ (Ref. 16) exchangecorrelation energies that are close to the recently published, to date most accurate, inhomogeneous Singwi-Tosi-LandSjölander energies. ${ }^{17}$

The nonspin-polarized total AM05 exchange-correlation energy, $E_{x c}^{\mathrm{AM} 05}$, is

$$
\begin{aligned}
E_{x c}^{\mathrm{AM} 05} & =\int n(\mathbf{r}) \epsilon_{x c}^{\mathrm{AM} 05}(n(\mathbf{r}), s(\mathbf{r})) d \mathbf{r}, \\
& =\int f_{x c}^{\mathrm{AM} 05}(n(\mathbf{r}), s(\mathbf{r})) d \mathbf{r} .
\end{aligned}
$$

We note that the functional, or the exchange-correlation energy per particle, $\epsilon_{x c}$, in each point is multiplied by the density in this point to obtain the exchange-correlation energy density, $f_{x c}$.

The exchange-correlation energy densities obtained from the separate subsystem functionals in Eqs. (1) and (2) can unambiguously be made spin-resolved by using the spin scaling for the total exchange energy:

$$
E_{x}\left[n_{\uparrow}, n_{\downarrow}\right]=\frac{1}{2}\left(E_{x}\left[2 n_{\uparrow}\right]+E_{x}\left[2 n_{\downarrow}\right]\right),
$$

and replacing the nonspin PW LDA correlation density with its spin-resolved form, $f_{c}^{\mathrm{LDA}}\left(n_{\uparrow}, n_{\downarrow}\right)=\left(n_{\uparrow}+n_{\downarrow}\right) \epsilon_{c}^{\mathrm{LDA}}\left(n_{\uparrow}, n_{\downarrow}\right)$. However, a slight ambiguity is introduced by the interpolation index, a unique feature of AM05, which necessitate a more elaborate exploration than is usual for GGAs in general.
If one lets the mixing between the spin-resolved versions of the interior and edge subsystem functionals still be determined by the total density and its scaled gradient via $s$ $=s(n,|\nabla n|)$ and $X(s)$, as in Eq. (5), one obtains a version we will refer to as $\boldsymbol{x t c t t}$. This and other upcoming acronyms are constructed to indicate whether the total or separate spin densities determine the interpolation index for exchange ${ }^{-}$and correlation while the last letter indicates if the translation from correlation energy per particle to correlation energy density is via total or spin densities. The $x \boldsymbol{t c t t}$ thus denotes that the total density determines the interpolation for both exchange and correlation, and the correlation energy per particle is multiplied by the total density to obtain the correlation energy density:

$$
\begin{aligned}
f_{x c}^{x t c t t}\left(n_{\uparrow}, n_{\downarrow}, s_{\uparrow}, s_{\downarrow}, s\right) \\
=\left\{\frac{1}{2}\left[f_{x}^{\mathrm{LDA}}\left(2 n_{\uparrow}\right)+f_{x}^{\mathrm{LDA}}\left(2 n_{\downarrow}\right)\right]+f_{c}^{\mathrm{LDA}}\left(n_{\uparrow}, n_{\downarrow}\right)\right\} X(s) \\
+\left\{\frac{1}{2}\left[f_{x}^{\mathrm{LAA}}\left(2 n_{\uparrow}, s_{\uparrow}\right)+f_{x}^{\mathrm{LAA}}\left(2 n_{\downarrow}, s_{\downarrow}\right)\right]+\gamma f_{c}^{\mathrm{LDA}}\left(n_{\uparrow}, n_{\downarrow}\right)\right\} \\
\\
\times[1-X(s)],
\end{aligned}
$$

where $f_{x}^{\mathrm{LDA}}(n)=n \epsilon_{x}^{\mathrm{LDA}}(n), f_{x}^{\mathrm{LAA}}(n, s)=n \epsilon_{x}^{\mathrm{LAA}}(n, s)$, and, using Eq. (3), we have defined

$$
s_{\nu}=s\left(2 n_{\nu},\left|\nabla\left(2 n_{\nu}\right)\right|\right) \quad \nu=\uparrow, \downarrow .
$$

However, this version leads to a total AM05 exchange energy that does not obey the exact exchange spin-scaling relation, Eq. (8).

A version of spin-polarized AM05 that obeys the exchange spin-scaling relation can be created by instead using separate indices for the spin-up $\left[X\left(s_{\uparrow}\right)\right]$ and spin-down $\left[X\left(s_{\downarrow}\right)\right]$ exchange energy densities. This is the $x \boldsymbol{s c t t}$ version (exchange interpolation is determined from the separate spin densities):

$$
\begin{aligned}
f_{x c}^{x s c t t}\left(n_{\uparrow}, n_{\downarrow}, s_{\uparrow}, s_{\downarrow}, s\right)= & \frac{1}{2}\left[f_{x}^{\mathrm{LDA}}\left(2 n_{\uparrow}\right) X\left(s_{\uparrow}\right)+f_{x}^{\mathrm{LDA}}\left(2 n_{\downarrow}\right) X\left(s_{\downarrow}\right)\right]+f_{c}^{\mathrm{LDA}}\left(n_{\uparrow}, n_{\downarrow}\right) X(s) \\
& +\frac{1}{2}\left\{f_{x}^{\mathrm{LAA}}\left(2 n_{\uparrow}, s_{\uparrow}\right)\left[1-X\left(s_{\uparrow}\right)\right]+f_{x}^{\mathrm{LAA}}\left(2 n_{\downarrow}, s_{\downarrow}\right)\left[1-X\left(s_{\downarrow}\right)\right]\right\}+\gamma f_{c}^{\mathrm{LDA}}\left(n_{\uparrow}, n_{\downarrow}\right)[1-X(s)] .
\end{aligned}
$$

The xsctt version of AM05 fulfills the spin-scaling relation, Eq. (8), but violates a fundamental principle of the subsystem functional scheme, the compatibility between exchange and correlation. Compatibility in this context requires that in each point in a system the exchange energy density needs to be combined with a correlation energy density based on the same model system as exchange. Since the amount of interior vs edge exchange and correlation in the xsctt version is determined with different indices, the total contribution in a point is not derived from the same model system. To restore the compatibility we need to use the same indices we use for exchange also for correlation. This version is $\boldsymbol{x s c s t}$ :

$$
\begin{aligned}
f_{x c}^{x s c s t}\left(n_{\uparrow}, n_{\downarrow}, s_{\uparrow}, s_{\downarrow}\right)= & \left(n_{\uparrow} \epsilon_{x}^{\mathrm{LDA}}\left(2 n_{\uparrow}\right)+\frac{n_{\uparrow}+n_{\downarrow}}{2} \epsilon_{c}^{\mathrm{LDA}}\left(n_{\uparrow}, n_{\downarrow}\right)\right) X\left(s_{\uparrow}\right)+\left(n_{\uparrow} \epsilon_{x}^{\mathrm{LAA}}\left(2 n_{\uparrow}, s_{\uparrow}\right)+\frac{n_{\uparrow}+n_{\downarrow}}{2} \gamma \epsilon_{c}^{\mathrm{LDA}}\left(n_{\uparrow}, n_{\downarrow}\right)\right)\left[1-X\left(s_{\uparrow}\right)\right] \\
& +\left(n_{\downarrow} \epsilon_{x}^{\mathrm{LDA}}\left(2 n_{\downarrow}\right)+\frac{n_{\uparrow}+n_{\downarrow}}{2} \epsilon_{c}^{\mathrm{LDA}}\left(n_{\uparrow}, n_{\downarrow}\right)\right) X\left(s_{\downarrow}\right)+\left(n_{\downarrow} \epsilon_{x}^{\mathrm{LAA}}\left(2 n_{\downarrow}, s_{\downarrow}\right)+\frac{n_{\uparrow}+n_{\downarrow}}{2} \gamma \epsilon_{c}^{\mathrm{LDA}}\left(n_{\uparrow}, n_{\downarrow}\right)\right)\left[1-X\left(s_{\downarrow}\right)\right] .
\end{aligned}
$$


Since the two separate interpolation indices used in xscst are constructed from the respective spin-up and spin-down densities, the extent to which the physics in a specific point is interpreted as edgelike or interiorlike is in the general case different for the two spin channels, i.e., $X\left(s_{\uparrow}\right) \neq X\left(s_{\downarrow}\right)$. A physical motivation for such separate environments over the joint treatment in $x t c t t$ is seen in, e.g., a strongly magnetic situation where one should expect the surface that confines spin-up and spin-down electrons to be placed differently.

The separate indices introduce a correlation energy per particle that is not the same for the spin-up and spin-down electrons, reflecting the possibility that spin-up and spindown electrons in a point actually can experience different physical environments: the spin-up electrons could be at a surface while the spin-down electrons could be freely moving as in an interior region. This is different from the case of most other functionals where the correlation energy per particle is usually determined by the total density and the relative spin polarization in a point, resulting in spin-up and spin-down particles contributing the same correlation energy per particle to the correlation energy density and the total correlation energy. The compatibility foundation of the subsystem functional scheme thus makes it natural to instead translate the correlation energy per particle into a correlation energy density by multiplying the separate spin-up and spindown parts of the correlation energy per particle with the spin-up/spin-down densities, respectively. We call this version for xscss:

$$
\begin{aligned}
& f_{x c}^{x s c s s}\left(n_{\uparrow}, n_{\downarrow}, s_{\uparrow}, s_{\downarrow}\right) \\
&=\left[n_{\uparrow} \epsilon_{x}^{\mathrm{LDA}}\left(2 n_{\uparrow}\right)+n_{\uparrow} \epsilon_{c}^{\mathrm{LDA}}\left(n_{\uparrow}, n_{\downarrow}\right)\right] X\left(s_{\uparrow}\right) \\
&+\left[n_{\uparrow} \epsilon_{x}^{\mathrm{LAA}}\left(2 n_{\uparrow}, s_{\uparrow}\right)+n_{\uparrow} \gamma \epsilon_{c}^{\mathrm{LDA}}\left(n_{\uparrow}, n_{\downarrow}\right)\right]\left[1-X\left(s_{\uparrow}\right)\right] \\
&+\left[n_{\downarrow} \epsilon_{x}^{\mathrm{LDA}}\left(2 n_{\downarrow}\right)+n_{\downarrow} \epsilon_{c}^{\mathrm{LDA}}\left(n_{\uparrow}, n_{\downarrow}\right)\right] X\left(s_{\downarrow}\right) \\
&+\left[n_{\downarrow} \epsilon_{x}^{\mathrm{LAA}}\left(2 n_{\downarrow}, s_{\downarrow}\right)+n_{\downarrow} \gamma \epsilon_{c}^{\mathrm{LDA}}\left(n_{\uparrow}, n_{\downarrow}\right)\right]\left[1-X\left(s_{\downarrow}\right)\right] .
\end{aligned}
$$

By considering a fully spin-polarized system, that is, a system where one of the spin densities is zero everywhere, we can further clarify this argument. In such a system the total correlation energy should be determined only by the nonzero spin density profile and the nonzero spin density based interpolation. However, since the correlation energy per particle is nonvanishing also for the nonexisting electrons, in xscst [Eq. (12)], the multiplication with the total density will give a nondesired contribution to the total correlation energy while in xscss [Eq. (13)] the multiplication with the zero density will result in no contribution. The xscss version is the final version of the AM05 spin functional and the arguments based on fundamental subsystem functional principles given here for this choice over other possible choices resolve the apparent ambiguity of how to handle the interpolation index in a spin-resolved version of AM05.

As seen in Eq. (13), the spin AM05 functional consists of a subsystem functional for interior regions and a subsystem functional for edge regions. The spin-up density and spindown density form separate density profiles, and separate indices are used for the separate density profiles to determine the ratio of interior vs edge functional to use.

Using that $\epsilon_{x}^{\mathrm{LAA}}(n, s)=\epsilon_{x}^{\mathrm{LDA}}(n) F_{x}^{\mathrm{LAA}}(s)$ (see Ref. 5), the AM05 exchange-correlation energy density in Eq. (13) can be written on the form

$$
\begin{aligned}
f_{x c}^{\mathrm{AM} 05} & \left(n_{\uparrow}, n_{\downarrow},\left|\nabla n_{\uparrow}\right|,\left|\nabla n_{\downarrow}\right|\right) \\
= & n_{\uparrow} \epsilon_{x}^{\mathrm{LDA}}\left(2 n_{\uparrow}\right) H_{x}\left(s_{\uparrow}\right)+n_{\downarrow} \epsilon_{x}^{\mathrm{LDA}}\left(2 n_{\downarrow}\right) H_{x}\left(s_{\downarrow}\right) \\
& +n_{\uparrow} \epsilon_{c}^{\mathrm{LDA}}\left(n_{\uparrow}, n_{\downarrow}\right) H_{c}\left(s_{\uparrow}\right)+n_{\downarrow} \epsilon_{c}^{\mathrm{LDA}}\left(n_{\uparrow}, n_{\downarrow}\right) H_{c}\left(s_{\downarrow}\right),
\end{aligned}
$$

where

$$
\begin{gathered}
H_{x}(s)=X(s)+[1-X(s)] F_{x}^{\mathrm{LAA}}(s), \\
H_{c}(s)=X(s)+[1-X(s)] \gamma .
\end{gathered}
$$

\section{IMPLEMENTING AM05}

It is straightforward to implement the AM05 exchangecorrelation energy directly from Ref. 5 and Eq. (14), the only minor obstacle being the need for a subroutine for calculating the Lambert W function ${ }^{18}$ used in $F_{x}^{\mathrm{LAA}}(s)$. At the AM05 website $^{19}$ a subroutine for calculating the AM05 exchangecorrelation energy is provided (including subroutines for the required Lambert $\mathrm{W}$ function and the LSDA correlation in the parametrization of Perdew and Wang ${ }^{12,13}$ ). The 35 line subroutine for the Lambert $\mathrm{W}$ function is also provided as supplemental material to this paper. ${ }^{20}$

In this section and Appendix B, however, we will explicitly give all formulas needed for implementing AM05, and for understanding the subroutines we provide at the AM05 website. ${ }^{19}$ In particular, we will give all necessary formulas for the exchange-correlation potentials needed for selfconsistent DFT implementations.

The most elaborate part of an AM05 implementation is the LAA refinement factor, $F_{x}^{\mathrm{LAA}}(s)$. From its definition in Eq. 8 in Ref. 5 we obtain

$$
\begin{gathered}
F_{x}^{\mathrm{LAA}}(s)=\frac{c s^{2}+1}{D(s)} \\
D(s)=c s A(s)+1 \\
A(s)=\frac{3}{\pi} \widetilde{\zeta}(s)^{1 / 2}\left\{\left[\left(\frac{4}{3}\right)^{1 / 3} \frac{2 \pi}{3}\right]^{4} \widetilde{\zeta}(s)^{2}+\widetilde{\zeta}(s)^{4}\right\} \\
=2\left(\frac{4}{3}\right)^{1 / 3} \widetilde{\zeta}(s)\left\{1+\left[\frac{27}{32 \pi^{2}}\right]^{2}\left[2\left(\frac{4}{3}\right)^{1 / 3} \widetilde{\zeta}(s)\right]^{2}\right\}^{1 / 4} \\
=Z(s)\left\{1+[k Z(s)]^{2}\right\}^{1 / 4}, \quad \text { where } k=\frac{27}{32 \pi^{2}}, \\
\widetilde{\zeta}(s)=\left[\frac{3}{2} W\left(\frac{s^{3 / 2}}{2 \sqrt{6}}\right)\right]^{2 / 3}=\frac{1}{2}\left(\frac{3}{4}\right)^{1 / 3} Z(s), \\
Z(s)=s\left[\frac{W(\chi(s))}{\chi(s)}\right]^{2 / 3}=\operatorname{constant} \times[W(\chi(s))]^{2 / 3},
\end{gathered}
$$




$$
\chi(s)=\frac{s^{3 / 2}}{2 \sqrt{6}},
$$

where $c=0.7168$, and $Z(s)$ is a normalized function (it is easy to verify that $Z(s) / s \rightarrow 1$ when $s \rightarrow 0$ ) defined by Eq. (21) which contains the Lambert $\mathrm{W}$ function and that it is convenient to use in implementations of AM05.

However, in self-consistent DFT calculations, not only is the exchange-correlation energy density needed but also the exchange-correlation potential for the spin-up electrons, the functional derivative of the exchange-correlation energy with respect to spin-up density, and its spin-down counter part:

$$
V_{x c, \nu}=\frac{\delta E_{x c}}{\delta n_{\nu}} \quad \nu=\uparrow, \downarrow .
$$

There are several different schemes for calculating the exchange-correlation potentials within a code, and in this section we will give the formulas needed for the White and
Bird $^{21}$ scheme, and the fully assembled exchange-correlation potentials (that we call the traditional scheme). A short overview of the different schemes for calculating the exchangecorrelation potentials, including information on how to transfer the White and Bird expressions here given into the input needed for the Pople, Gill, and Johnson ${ }^{22}$ scheme, and detailed derivations, applicable also for other GGA-type functionals, are given in Appendix B.

Generally for a GGA-type functional the White and Bird scheme needs the five quantities $\frac{\partial f_{x c}\left(n_{\uparrow}, n_{\downarrow},\left|\nabla n_{\uparrow}\right|,\left|\nabla n_{\downarrow},\right|, \nabla n \mid\right)}{\partial n_{\uparrow}}$, $\frac{\partial f_{x c}\left(n_{\uparrow}, n_{\downarrow},\left|\nabla n_{\uparrow}\right|,\left|\nabla n_{\downarrow}\right|,|\nabla n|\right)}{\partial n_{\downarrow}}, \quad \frac{\partial f_{x c}\left(n_{\uparrow}, n_{\downarrow},\left|\nabla n_{\uparrow}\right|,\left|\nabla n_{\downarrow}\right|,|\nabla n|\right)}{\partial\left|\nabla n_{\uparrow}\right|}$, $\frac{\partial f_{x c}\left(n_{\uparrow}, n_{\downarrow},\left|\nabla n_{\uparrow}\right|,\left|\nabla n_{\downarrow}\right|,|\nabla n|\right)}{\partial\left|\nabla n_{\downarrow}\right|}$, and $\frac{\partial f_{x c}\left(n_{\uparrow}, n_{\downarrow},\left|\nabla n_{\uparrow}\right|,\left|\nabla n_{\downarrow}\right|,|\nabla n|\right)}{\partial|\nabla n|}$. Since AM05 does not use $|\nabla n|$, we immediately see that

$$
\frac{\partial f_{x c}^{\mathrm{AM} 05}\left(n_{\uparrow}, n_{\uparrow},\left|\nabla n_{\uparrow}\right|,\left|\nabla n_{\downarrow}\right|\right)}{\partial|\nabla n|}=0 .
$$

The remaining four quantities are

$$
\begin{aligned}
\frac{\partial f_{x c}^{\mathrm{AM} 05}\left(n_{\uparrow}, n_{\uparrow},\left|\nabla n_{\uparrow}\right|,\left|\nabla n_{\downarrow}\right|\right)}{\partial n_{\nu_{a}}}= & v_{x}^{\mathrm{LDA}}\left(2 n_{\nu_{a}}\right) H_{x}\left(s_{\nu_{a}}\right)-\frac{4}{3} \epsilon_{x}^{\mathrm{LDA}}\left(2 n_{\nu_{a}}\right) s_{\nu_{a}} \frac{\partial H_{x}\left(s_{\nu_{a}}\right)}{\partial s_{\nu_{a}}}+v_{c, \nu_{a}}^{\mathrm{LDA}}\left(n_{\uparrow}, n_{\downarrow}\right) \frac{1}{\left(n_{\uparrow}+n_{\downarrow}\right)}\left[n_{\uparrow} H_{c}\left(s_{\uparrow}\right)+n_{\downarrow} H_{c}\left(s_{\downarrow}\right)\right] \\
& +\epsilon_{c}^{\mathrm{LDA}}\left(n_{\uparrow}, n_{\downarrow}\right) \frac{n_{\nu_{b}}}{\left(n_{\uparrow}+n_{\downarrow}\right)}\left[H_{c}\left(s_{\nu_{a}}\right)-H_{c}\left(s_{v_{b}}\right)\right]-\frac{4}{3} \epsilon_{c}^{\mathrm{LDA}}\left(n_{\uparrow}, n_{\downarrow}\right) s_{v_{a}} \frac{\partial H_{c}\left(s_{\nu_{a}}\right)}{\partial s_{v_{a}}},
\end{aligned}
$$

and

$$
\frac{\partial f_{x c}^{\mathrm{AM} 05}\left(n_{\uparrow}, n_{\uparrow},\left|\nabla n_{\uparrow}\right|,\left|\nabla n_{\downarrow}\right|\right)}{\partial\left|\nabla n_{\nu_{a}}\right|}=\frac{\epsilon_{x}^{\mathrm{LDA}}\left(2 n_{\nu_{a}}\right)}{2 k_{F, \nu_{a}}} \frac{\partial H_{x}\left(s_{\nu_{a}}\right)}{\partial s_{\nu_{a}}}+\frac{\epsilon_{c}^{\mathrm{LDA}}\left(n_{\uparrow}, n_{\downarrow}\right)}{2 k_{F, \nu_{a}}} \frac{\partial H_{c}\left(s_{\nu_{a}}\right)}{\partial s_{\nu_{a}}},
$$

where $\nu_{a}=\uparrow$ or $\downarrow$, and $\nu_{b}$ denotes the opposite spin to $\nu_{a}$. We have defined $k_{F, \nu}=k_{F}\left(2 n_{\nu}\right)$, and $v_{x}^{\mathrm{LDA}}\left(2 n_{\nu}\right)$ is the LDA exchange potential, and $v_{c, \nu}^{\mathrm{LDA}}\left(n_{\uparrow}, n_{\downarrow}\right)$ is the LDA correlation potential for the $\nu$ spin. The explicit formulas for the derivatives of $H_{x}(s)$ and $H_{c}(s)$ are given in Appendix B.

In the traditional scheme, in addition to $s_{\nu}, \nu=\uparrow, \downarrow$, given in Eq. (10), four other dimensionless density derivatives are used:

$$
t_{\nu}=\frac{\nabla^{2}\left(2 n_{\nu}\right)}{\left(2 k_{F, \nu}\right)^{2}\left(2 n_{\nu}\right)} \quad \text { and } \quad u_{\nu}=\frac{\nabla\left(2 n_{\nu}\right) \cdot \nabla\left|\nabla\left(2 n_{\nu}\right)\right|}{\left(2 k_{F, \nu}\right)^{3}\left(2 n_{\nu}\right)^{2}} .
$$

The full explicit exchange-correlation potentials are

$$
\begin{aligned}
& V_{x c, \nu_{a}}^{\mathrm{AM} 05}=v_{x}^{\mathrm{LDA}}\left(2 n_{v_{a}}\right)\left[H_{x}\left(s_{\nu_{a}}\right)-s_{v_{a}} \frac{\partial H_{x}\left(s_{\nu_{a}}\right)}{\partial s_{v_{a}}}\right]+\epsilon_{x}^{\mathrm{LDA}}\left(2 n_{v_{a}}\right)\left\{\left(\frac{4}{3} s_{v_{a}}^{2}-t_{v_{a}}\right) \frac{1}{s_{\nu_{a}}} \frac{\partial H_{x}\left(s_{v_{a}}\right)}{\partial s_{v_{a}}}+\left(\frac{4}{3} s_{\nu_{a}}^{3}-u_{v_{a}}\right) \frac{\partial}{\partial s_{v_{a}}}\left[\frac{1}{s_{v_{a}}} \frac{\partial H_{x}\left(s_{v_{a}}\right)}{\partial s_{\nu_{a}}}\right]\right\} \\
& +v_{c, \nu_{a}}^{\mathrm{LDA}}\left(n_{\uparrow}, n_{\downarrow}\right)\left(H_{c}\left(s_{v_{a}}\right)-s_{\nu_{a}} \frac{\partial H_{c}\left(s_{\nu_{a}}\right)}{\partial s_{\nu_{a}}}\right)+\epsilon_{c}^{\mathrm{LDA}}\left(n_{\uparrow}, n_{\downarrow}\right)\left\{\left(\frac{4}{3} s_{v_{a}}^{2}-t_{\nu_{a}}\right) \frac{1}{s_{\nu_{a}}} \frac{\partial H_{c}\left(s_{v_{a}}\right)}{\partial s_{\nu_{a}}}+\left(\frac{4}{3} s_{v_{a}}^{3}-u_{\nu_{a}}\right) \frac{\partial}{\partial s_{\nu_{a}}}\left[\frac{1}{s_{v_{a}}} \frac{\partial H_{c}\left(s_{\nu_{a}}\right)}{\partial s_{v_{a}}}\right]\right\} \\
& +\left[\epsilon_{c}^{\mathrm{LDA}}\left(n_{\uparrow}, n_{\downarrow}\right)-v_{c, \nu_{a}}^{\mathrm{LDA}}\left(n_{\uparrow}, n_{\downarrow}\right)\right] \frac{n_{\nu_{b}}}{n_{\uparrow}+n_{\downarrow}}\left[H_{c}\left(s_{\nu_{a}}\right)-H_{c}\left(s_{\nu_{b}}\right)-s_{\nu_{a}} \frac{\partial H_{c}\left(s_{\nu_{a}}\right)}{\partial s_{\nu_{a}}}\right] \\
& +\left[\epsilon_{c}^{\mathrm{LDA}}\left(n_{\uparrow}, n_{\downarrow}\right)-v_{c, \nu_{b}}^{\mathrm{LDA}}\left(n_{\uparrow}, n_{\downarrow}\right)\right] \frac{\nabla n_{\uparrow} \cdot \nabla n_{\downarrow}}{\left|\nabla n_{\nu_{a}}\right|^{2}} \frac{n_{\nu_{a}}}{n_{\uparrow}+n_{\downarrow}} s_{\nu_{a}} \frac{\partial H_{c}\left(s_{\nu_{a}}\right)}{\partial s_{\nu_{a}}} .
\end{aligned}
$$


Iron: AM05 (xscss)

on PBE (full) and LDA (dashed) PAWpotentials

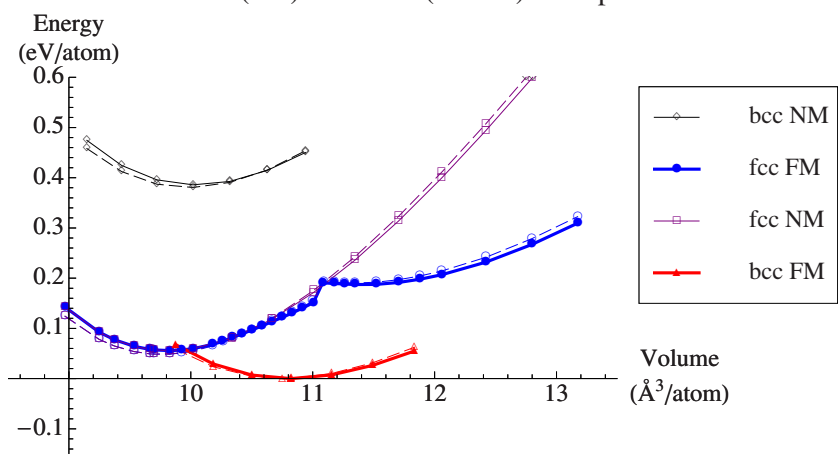

FIG. 1. (Color online) Energy vs volume for non-magnetic (NM) and ferro-magnetic (FM) phases of bcc and fcc iron, calculated with AM05.

Again, $\nu_{a}=\uparrow$ or $\downarrow$, and $\nu_{b}$ denotes the opposite spin to $\nu_{a}$. A detailed derivation of Eq. (28), and formulas for the derivatives of $H_{x}(s)$ and $H_{c}(s)$, are given in Appendix B. Note that even though the AM05 functional itself is not dependent on the total density gradient, this gradient is indeed present in the full correlation potential via the quantity $\nabla n_{\uparrow} \cdot \nabla n_{\downarrow}$ $=\left(|\nabla n|^{2}-\left|\nabla n_{\uparrow}\right|^{2}-\left|\nabla n_{\uparrow}\right|^{2}\right) / 2$. This have no bearing on the implementation of AM05 since the magnitude of the gradient of the total density is used in ordinary PBE (Ref. 9) correlation while the magnitude of the gradients of the separate spin-up and spin-down densities are used in PBE exchange. As is the case with AM05 potentials obtained by the White and Bird, and the Pople, Gill, and Johnson schemes, AM05 potentials via the traditional scheme can thus be readily implemented in every code already containing PBE. Note that if exchange and correlation are treated separately in a code, it could be more convenient to include the last part of second line in Eq. (28) into the exchange part than the correlation part since ordinary GGA exchange [see last part of first line in Eq. (28)] already uses the needed density derivative quantities $t_{\nu}$ and $u_{\nu}$.

\section{AM05 RESULTS FOR Fe}

We have investigated the spin version of AM05 by performing nonspinresolved and spin-resolved calculations for

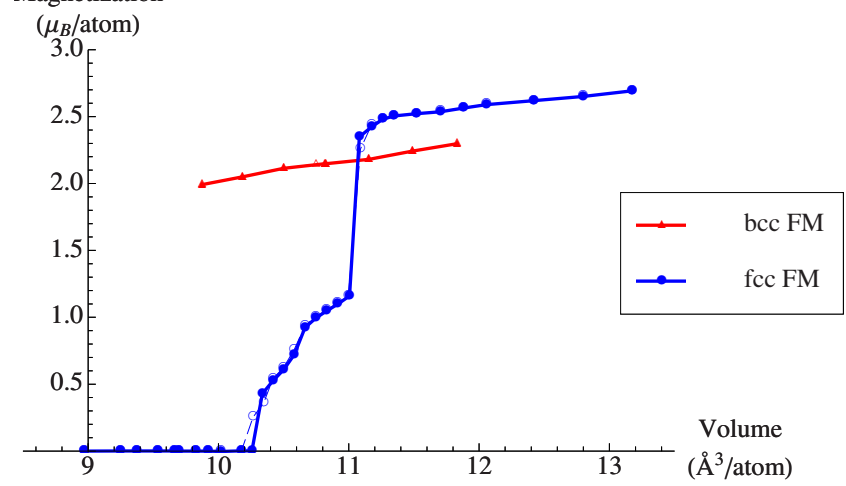

FIG. 2. (Color online) Magnetization vs volume for the FM phases of bcc and fcc iron, calculated with AM05.
Iron: Comparison between AM05 (xscss) and xsctt, xscst, xtctt, (from bottom to top) on PBE PAW potentials

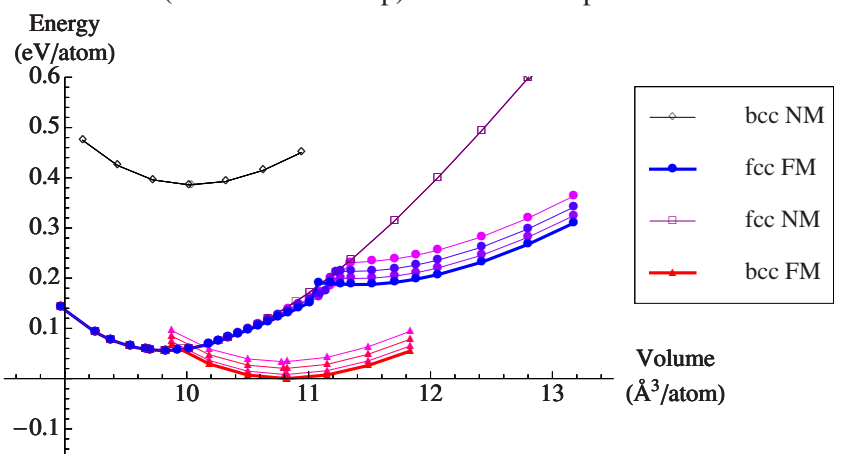

FIG. 3. (Color online) Energy vs volume for NM and FM phases of bcc and fcc iron, calculated with the AM05 functional and three intermediate spin versions. All spin versions give the same results when the magnetization is zero, when they all collapse to the unique nonspin version of AM05.

the bec and fcc phases of iron, using VASP. ${ }^{23}$ As is described in Refs. 6 and 24, in VASP 5 existing projector augmented wave (PAW) potentials can be used together with functionals they are not created with. Note that this is not generally true for other implementations of the PAW potentials and other types of pseudopotentials but must be tested thoroughly from case to case. Further details of our calculations are given in Appendix A.

Figures 1 and 2 show that minimal differences are obtained in AM05 results using LDA or PBE PAW potentials. It is clearly seen that AM05 obtains the correct ground state, the ferromagnetic bcc phase. Interestingly also the differences between the four different spin versions are very small, as seen in Figs. 3 and 4. In particular, there are no differences in lattice constants, bulk moduli, and magnetizations, in the bcc ground state, see Table I. The main difference is instead the energy difference between states with widely different magnetizations, such as nonmagnetic fcc and magnetic bcc, a property that is hard to derive from experiments. This will be investigated and discussed in future publications.

Iron: Comparison between AM05 (xscss) and xsctt, xscst, xtctt,

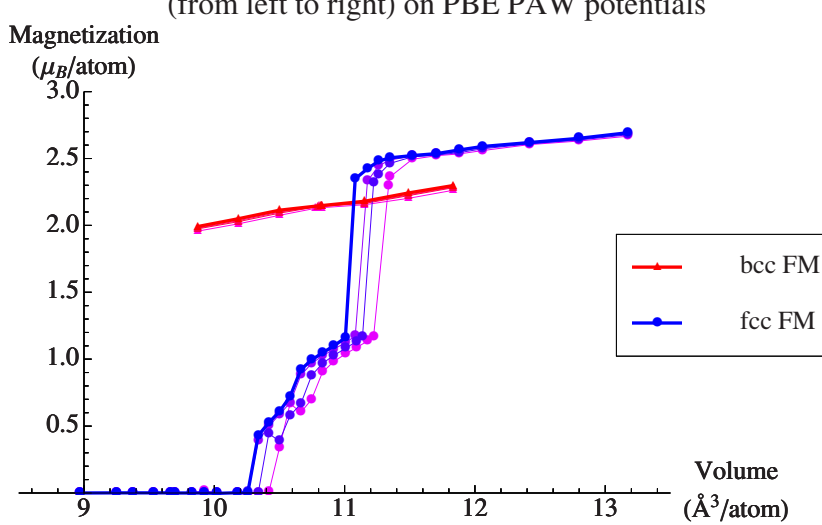

FIG. 4. (Color online) Magnetization vs volume for the FM phases of bcc and fcc iron, calculated with the AM05 functional and three intermediate spin versions. 
TABLE I. Lattice constants, bulk moduli, and magnetizations, for the ground-state bcc phase, obtained with the spin version of $\mathrm{AM} 05$, and the three intermediate versions. Experimental values are taken from Ref. 25.

\begin{tabular}{lccc}
\hline \hline Version & $\begin{array}{c}\text { Lattice constant } \\
(\AA)\end{array}$ & $\begin{array}{c}\text { Bulk modulus } \\
(\mathrm{GPa})\end{array}$ & $\begin{array}{c}\text { Magnetization } \\
\left(\mu_{B}\right)\end{array}$ \\
\hline xtctt & 2.783 & 222 & 2.127 \\
xscst & 2.784 & 220 & 2.140 \\
xsctt & 2.785 & 220 & 2.143 \\
AM05 (xscss) & 2.786 & 218 & 2.148 \\
Experiment & 2.86 & 168 & 2.22 \\
PBE & 2.83 & 185 & 2.20 \\
\hline \hline
\end{tabular}

Finally, in Figs. 5 and 6 we compare the AM05 results with those obtained with LDA and the PBE (Ref. 9) functionals. AM05 gives the same correct ferromagnetic bcc ground state as PBE while LDA gives the wrong spin state. Thus, the inclusion of surface physics into AM05 corrects one of the largest deficiencies of LDA. However, the PBE lattice constant, bulk modulus, and magnetization are closer to the experimental values than the corresponding AM05 results, see Table I. This observation is further discussed in the next section.

\section{CONCLUSIONS}

We have discussed several possible versions of the spin density generalized AM05 functional. One of these versions, xscss, is found to extend the underlying subsystem functional framework of AM05 in a natural way, and is therefore preferred. As a benchmark calculation we have chosen the ground state of Fe. The different versions of spin AM05 perform very similarly and all give the correct spin ground state. Inclusion of surface physics into AM05 thus corrects a major failure of LDA, which gives the wrong spin state for this system. However, the lattice constants are not in as good agreement with experiment as PBE. Since nonspin AM05 generally is more accurate than PBE for nonmagnetic solidstate systems, ${ }^{6,8}$ there are two possible reasons for this. It might be that Fe just is a problematic case for AM05. But another possibility is that the excellent performance of AM05 for nonspin-polarized systems does not directly generalize to a similar performance for spin-resolved cases when using spin-AM05. Either due to that the underlying spin formulation of the more approximate correlation part of the Airy Gas based edge functional in Eq. (2) is not adequate. Or that magnetic materials are of a different class than nonmagnetic materials (containing more localized electrons), which the interior (uniform electron gas based) and edge (Airy gas surface system) subsystem functionals included in AM05 cannot adequately describe. The lattice constant and bulk moduli results of Ropo et al. ${ }^{7}$ indicate that the deficiency is not only for Fe but more general for $3 d$ metals. If so, the minimal differences in results between the different versions of AM05 discussed in Sec. II, shown in Figs. 3 and 4, and Table I, indicate that a change in the correlation of the edge

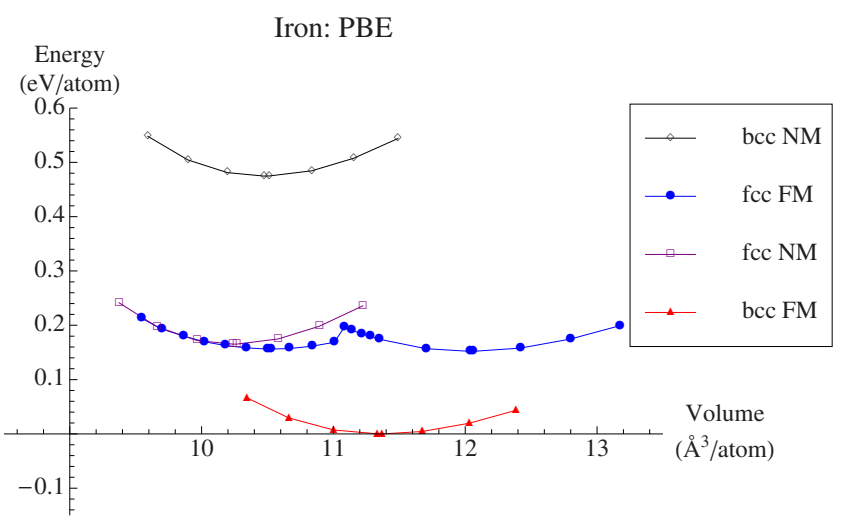

Iron: AM05(xscss) on

PBE PAW potentials
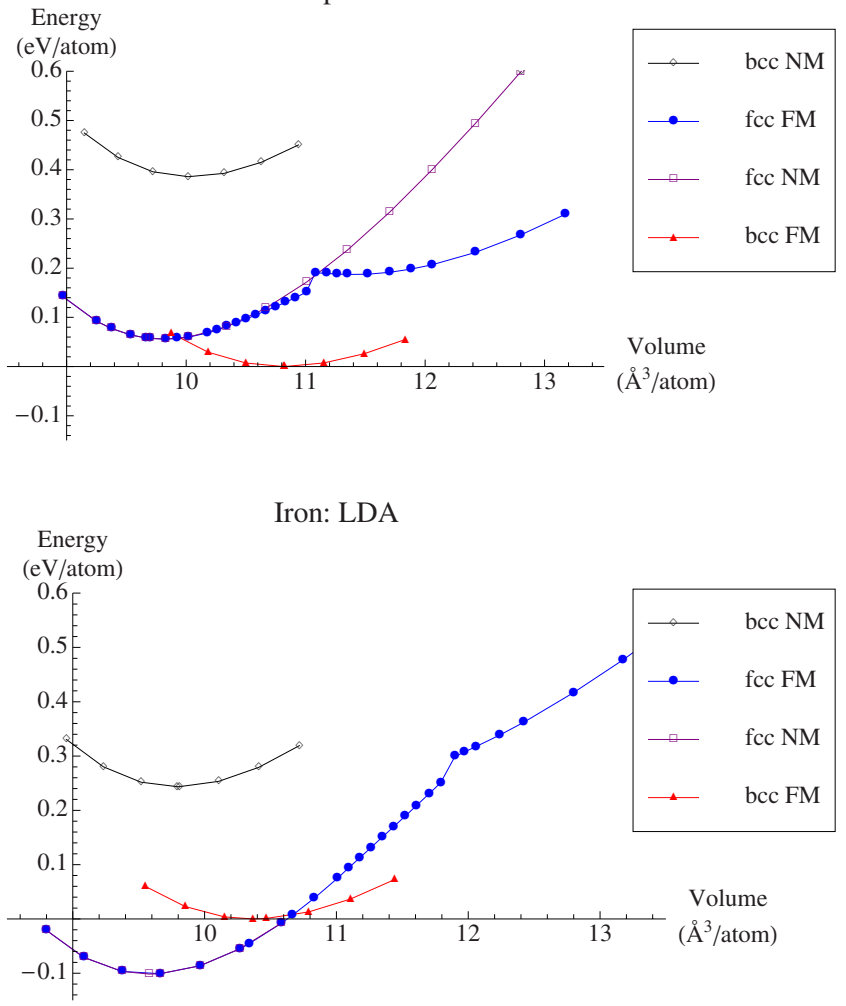

FIG. 5. (Color online) Energy vs volume for NM and FM phases of bec and fec iron, calculated using LDA, AM05, and PBE.

functional in Eq. (2) would not result in a large change in lattice constant, and thus not cure this deficiency. However, other properties than lattice constants, bulk moduli, and magnetizations should also be considered, in particular properties that more closely probe differences in energy between nonspin and spin phases of solids. The most striking difference between the PBE and AM05 results shown in Fig. 5 is the slope of the spin-resolved fcc curve, and properties probing this should be investigated. Future applications of spinAM05 will show which of the scenarios discussed above is the correct one. If AM05 turns out to generally be less accurate for spin-polarized systems than for nonspin-polarized systems, the next functional constructed according to the subsystem functional scheme should address this deficiency. 


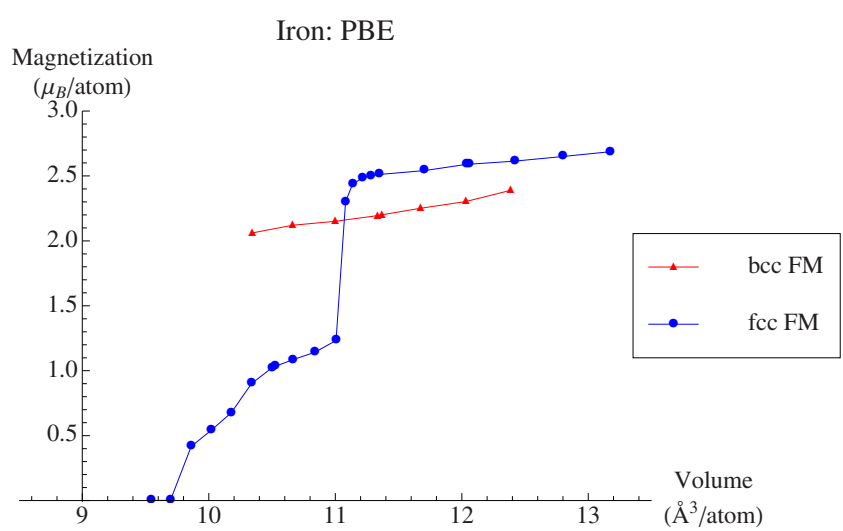

Iron: AM05 (xscss) on PBE PAW potentials
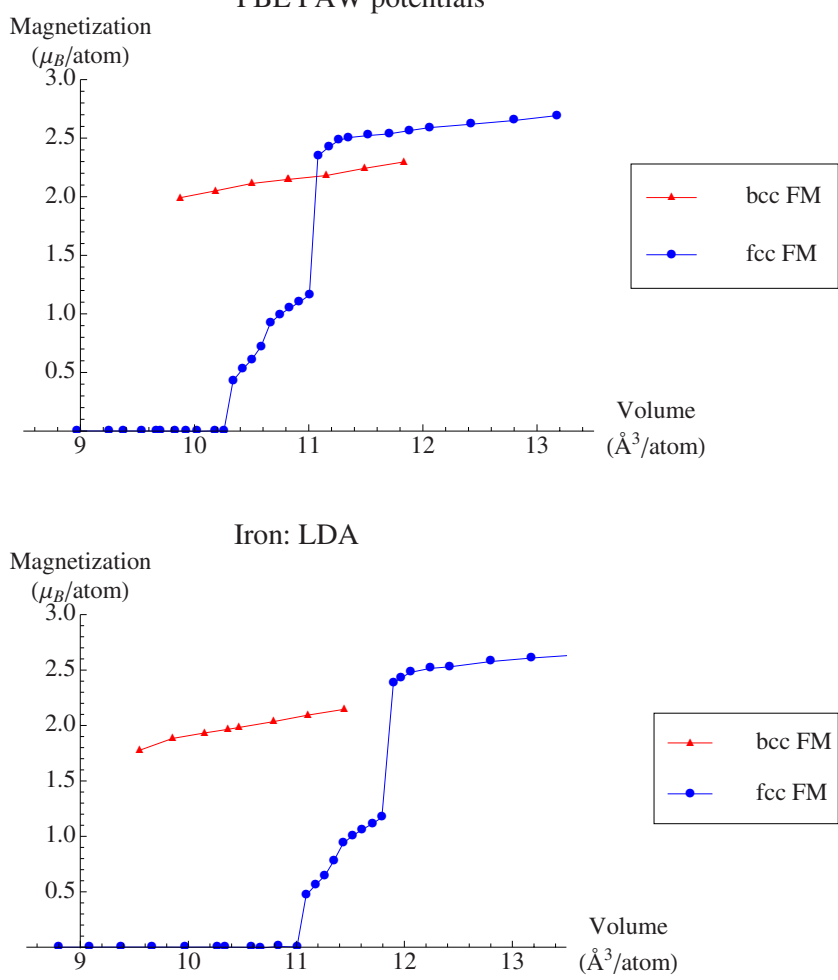

FIG. 6. (Color online) Magnetization vs volume for the FM phases of bec and fcc iron, calculated using LDA, AM05, and PBE.

\section{ACKNOWLEDGMENTS}

We thank T. R. Mattsson for fruitful discussions. R.A. acknowledges support from the Alexander von Humboldt Foundation and the German-Israeli Foundation. Sandia is a multiprogram laboratory operated by Sandia Corporation, a Lockheed Martin Company, for the United States Department of Energy's National Nuclear Security Administration under contract DE-AC04-94AL85000.

\section{APPENDIX A: DETAILS OF THE CALCULATIONS (CF. REF. 26)}

We implemented the spin version of AM05 and the three intermediate versions into version 5.1.40 of VASP, which al- ready had nonspin AM05 implemented. We used cubic cells for both the bcc (two atoms) and fcc (four atoms) phases, and $16 \times 16 \times 16 k$ points in the Monkhorst-Pack scheme. ${ }^{27}$ The PAW potentials are standard, the PBE one is labeled "PAW_PBE Fe 06Sep2000," and the LDA one "PAW Fe 03Mar1998." A cutoff energy of $350 \mathrm{eV}$ was used in all calculations. For the spin-resolved calculations an initial magnetic moment of $4 \mu_{B}$ on each site was used. The lattice constants and bulk moduli presented in Table I, and the corresponding PBE results are calculated by a fit of seven energy/volume pairs, spaced evenly $\pm 10 \%$ around the equilibrium volume, to the Murnaghan ${ }^{28}$ equation of state. The magnetization was subsequently calculated at the obtained minimum-energy point by a separate VASP 5 calculation that also served as a control so that the energy obtained in this point indeed was lower than in the previously calculated points. Our PBE results compares well with the PW91 results of Ref. 25, as do our PBE and xscst results for lattice constant and bulk moduli with those of Ropo et al. (Ref. 7). The calculations for some of the intermediate spin versions converged slowly in the range where the magnetization changes rapidly with lattice constant. The results in this region are sensitive to initial conditions; this can be seen in Fig. 4 in that some of the curves are jagged. Even though the magnetization in this region might be less accurate than in other regions, the general trend is not affected.

\section{APPENDIX B: IMPORTANT FORMULAS AND BACKGROUND INFORMATION FOR IMPLEMENTING SPIN AM05 INTO DFT CODES}

The exchange-correlation energy for a spin-polarized system can be written as

$$
E_{x c}=\int d \mathbf{r} f_{x c}\left(n_{\uparrow}(\mathbf{r}), n_{\downarrow}(\mathbf{r}), \nabla n_{\uparrow}(\mathbf{r}), \nabla n_{\downarrow}(\mathbf{r})\right),
$$

where $n_{\uparrow}(\mathbf{r})$ and $n_{\downarrow}(\mathbf{r})$ are the densities of spin-up electrons and spin-down electrons at $\mathbf{r}$, respectively, $d \mathbf{r}=d r_{x} d r_{y} d r_{z}$ is the volume element, and $f_{x c}=f_{x}+f_{c}$ is the exchangecorrelation energy density. $f_{x c}$ is generally a functional of the spin-up and spin-down densities, $f_{x c}=f_{x c}\left[n_{\uparrow}(\mathbf{r}), n_{\downarrow}(\mathbf{r})\right]$. We here restrict ourselves to the case where the functional dependency is only through the densities and gradients of the densities since this is the case for AM05 [see Eq. (14)] and most other GGA-type functionals.

In this appendix we will explicitly describe how to obtain the potentials given in Eq. (23), needed for self-consistent calculations. In addition, in some codes, the stress tensor is calculated and a functional routine also needs to output some ingredients for this calculation. The stress tensor is

$$
\begin{aligned}
\sigma_{\alpha \beta}= & \delta_{\alpha \beta} E_{x c}[n(\mathbf{r})]+\left.\sum_{\xi=\uparrow, \downarrow} \int d \mathbf{r} V_{x c, \xi}(\mathbf{r}) \frac{\partial n_{\xi}(\hat{\varepsilon}, \mathbf{r})}{\partial \varepsilon_{\alpha \beta}}\right|_{\hat{\varepsilon}=0} \\
& -\sum_{\xi=\uparrow, \downarrow} \int d \mathbf{r} \frac{\partial f_{x c}\left(n_{\uparrow}(\mathbf{r}), n_{\downarrow}(\mathbf{r}), \nabla n_{\uparrow}(\mathbf{r}), \nabla n_{\downarrow}(\mathbf{r})\right)}{\partial\left(\frac{\partial n_{\xi}(\mathbf{r})}{\partial r_{\beta}}\right)} \frac{\partial n_{\xi}(\mathbf{r})}{\partial r_{\alpha}} .
\end{aligned}
$$

The terms on the first line of this expression are handled as 
in any LDA calculation, only replacing $E_{x c}, V_{x c, \uparrow}$, and $V_{x c, \downarrow}$ with the corresponding GGA quantities. They will not be further discussed here. The terms on the second line constitutes a GGA correction and we will explain how to obtain the needed functional related ingredients for this correction.

The exchange-correlation potential, Eq. (23), for the exchange-correlation energy in Eq. (B1) is

$$
\begin{aligned}
V_{x c, \nu}(\mathbf{r})= & \frac{\partial f_{x c}\left(n_{\uparrow}(\mathbf{r}), n_{\downarrow}(\mathbf{r}), \nabla n_{\uparrow}(\mathbf{r}), \nabla n_{\downarrow}(\mathbf{r})\right)}{\partial n_{\nu}(\mathbf{r})} \\
& -\nabla \cdot \frac{\partial f_{x c}\left(n_{\uparrow}(\mathbf{r}), n_{\downarrow}(\mathbf{r}), \nabla n_{\uparrow}(\mathbf{r}), \nabla n_{\downarrow}(\mathbf{r})\right)}{\partial \nabla n_{\nu}(\mathbf{r})} .
\end{aligned}
$$

The first term in this potential is straightforward to obtain but the second term can be treated in several ways. In the traditional scheme this term is expanded until derivatives of the densities and derivatives of $f_{x c}$ are separated. This means that for a given density the full $V_{x c, \nu}$ can be calculated within the functional routine. In contrast, the White and Bird, and Pople, Gill, and Johnson schemes only partially expand this term and $V_{x c, \nu}$ needs to be assembled in a routine outside of the functional subroutine.

Let us focus on the second term in Eq. (B3) for the moment. It is a scalar product between two vector quantities, $\nabla$ and $\frac{\partial f_{x c}\left(n_{\uparrow}(\mathbf{r}), n_{\downarrow}(\mathbf{r}), \nabla n_{\uparrow}(\mathbf{r}), \nabla n_{\downarrow}(\mathbf{r})\right)}{\partial \nabla n_{\nu}(\mathbf{r})}$. In a plane-wave basis this second term is readily Fourier transformed:

$$
\begin{aligned}
\nabla \cdot & \frac{\partial f_{x c}\left(n_{\uparrow}(\mathbf{r}), n_{\downarrow}(\mathbf{r}), \nabla n_{\uparrow}(\mathbf{r}), \nabla n_{\downarrow}(\mathbf{r})\right)}{\partial \nabla n_{\nu}(\mathbf{r})} \\
= & \frac{1}{N} \sum_{\mathbf{G}, \mathbf{r}^{\prime}} i \mathbf{G} \cdot \frac{\partial f_{x c}\left(n_{\uparrow}\left(\mathbf{r}^{\prime}\right), n_{\downarrow}\left(\mathbf{r}^{\prime}\right), \nabla n_{\uparrow}\left(\mathbf{r}^{\prime}\right), \nabla n_{\downarrow}\left(\mathbf{r}^{\prime}\right)\right)}{\partial \nabla n_{\nu}\left(\mathbf{r}^{\prime}\right)} \\
& \times e^{i \mathbf{G} \cdot\left(\mathbf{r}-\mathbf{r}^{\prime}\right)},
\end{aligned}
$$

this is the White and Bird scheme. ${ }^{21}$

In quantum chemistry codes using finite basis sets $\phi_{\mu}$ $(\mu=1, \ldots, N)$, the Fock matrices are the needed objects and by integration by parts, one obtains

$$
\begin{aligned}
\int \nabla & \cdot \frac{\partial f_{x c}\left(n_{\uparrow}(\mathbf{r}), n_{\downarrow}(\mathbf{r}), \nabla n_{\uparrow}(\mathbf{r}), \nabla n_{\downarrow}(\mathbf{r})\right)}{\partial \nabla n_{\nu}(\mathbf{r})} \phi_{\mu} \phi_{\nu} d \mathbf{r} \\
& =-\int \frac{\partial f_{x c}\left(n_{\uparrow}(\mathbf{r}), n_{\downarrow}(\mathbf{r}), \nabla n_{\uparrow}(\mathbf{r}), \nabla n_{\downarrow}(\mathbf{r})\right)}{\partial \nabla n_{\nu}(\mathbf{r})} \cdot \nabla\left(\phi_{\mu} \phi_{\nu}\right) d \mathbf{r},
\end{aligned}
$$

this is the Pople, Gill, and Johnson scheme. ${ }^{22}$

It is obvious that since the two terms in Eq. (B3) are not treated in the same way in the White and Bird, and the Pople, Gill, and Johnson schemes, the exchange-correlation potential (or Fock matrices) needs to be assembled outside of a functional subroutine and that this subroutine, instead of the full $V_{x c, \uparrow}$ and $V_{x c, \downarrow}$, needs to output $\frac{\partial f_{x c}\left(n_{\uparrow}, n_{\downarrow}, \nabla n_{\uparrow}, \nabla n_{\downarrow}\right)}{\partial n_{\uparrow}}$ and $\frac{\partial f_{x c}\left(n_{\uparrow}, n_{\downarrow}, \nabla n_{\uparrow}, \nabla n_{\downarrow}\right)}{\partial \nabla n_{\uparrow}}$, and the corresponding spin-down quantities, where we from now on will omit the spatial argument of the densities.

However, $\frac{\partial f_{x c}\left(n_{\uparrow}, n_{\downarrow}, \nabla n_{\uparrow}, \nabla n_{\downarrow}\right)}{\partial \nabla n_{\uparrow}}$ is a vector and together with its spin-down counterpart gives a total of six different scalar quantities to calculate. Due to the fact that functionals of GGA type for symmetry reasons only depend on the gradients of the densities through their absolute values, $\left|\nabla n_{\uparrow}\right|$, $\left|\nabla n_{\downarrow}\right|$, and $|\nabla n|$, where $n=n_{\uparrow}+n_{\downarrow}$, only three scalar quantities are actually needed. Note that since $|\nabla n|^{2}=\left|\nabla n_{\uparrow}\right|^{2}+\left|\nabla n_{\downarrow}\right|^{2}$ $+2 \nabla n_{\uparrow} \cdot \nabla n_{\downarrow} \neq\left|\nabla n_{\uparrow}\right|^{2}+\left|\nabla n_{\downarrow}\right|^{2}$, the dependency on the two vector quantities $\nabla n_{\uparrow}$ and $\nabla n_{\downarrow}$, needs to be replaced by a dependency on three scalar quantities. It is customary to use $\left|\nabla n_{\uparrow}\right|,\left|\nabla n_{\uparrow}\right|$, and $|\nabla n|$ in the traditional and the White and Bird schemes while $\left|\nabla n_{\uparrow}\right|^{2},\left|\nabla n_{\downarrow}\right|^{2}$, and $\nabla n_{\uparrow} \cdot \nabla n_{\downarrow}$ are used in the Pople, Gill, and Johnson scheme.

One can show that

$$
\begin{gathered}
\frac{\partial f_{x c}\left(n_{\uparrow}, n_{\downarrow}, \nabla n_{\uparrow}, \nabla n_{\downarrow}\right)}{\partial \nabla n_{\nu_{a}}} \\
=2 \frac{\partial f_{x c}\left(n_{\uparrow}, n_{\downarrow},\left|\nabla n_{\uparrow}\right|^{2},\left|\nabla n_{\downarrow}\right|^{2}, \nabla n_{\uparrow} \cdot \nabla n_{\downarrow}\right)}{\partial\left|\nabla n_{\nu_{a}}\right|^{2}} \nabla n_{\nu_{a}} \\
\quad+\frac{\partial f_{x c}\left(n_{\uparrow}, n_{\downarrow},\left|\nabla n_{\uparrow}\right|^{2},\left|\nabla n_{\downarrow}\right|^{2}, \nabla n_{\uparrow} \cdot \nabla n_{\downarrow}\right)}{\partial \nabla n_{\uparrow} \cdot \nabla n_{\downarrow}} \nabla n_{\nu_{b}} \\
=\frac{\partial f_{x c}\left(n_{\uparrow}, n_{\downarrow},\left|\nabla n_{\uparrow}\right|,\left|\nabla n_{\downarrow}\right|,|\nabla n|\right)}{\partial\left|\nabla n_{\nu_{a}}\right|} \frac{\nabla n_{\nu_{a}}}{\left|\nabla n_{\nu_{a}}\right|} \\
\quad+\frac{\partial f_{x c}\left(n_{\uparrow}, n_{\downarrow},\left|\nabla n_{\uparrow}\right|,\left|\nabla n_{\downarrow}\right|,|\nabla n|\right)}{\partial|\nabla n|} \frac{\nabla n}{|\nabla n|},
\end{gathered}
$$

where the first equality [Eq. (B6)] gives the quantity that is usually seen in the Pople, Gill, and Johnson scheme and the second equality [Eq. (B7)] is seen in the White and Bird scheme, and $\nu_{a}=\uparrow$ or $\downarrow$, and $\nu_{b}$ denotes the opposite spin from $\nu_{a} \cdot \frac{\nabla n_{\uparrow}}{\left|\nabla n_{\uparrow}\right|}, \frac{\nabla n_{\downarrow}}{\left|\nabla n_{1}\right|}$, and $\frac{\nabla n}{|\nabla n|}$ are unit vectors that are well defined even when $\left|\nabla n_{\uparrow}\right|,\left|\nabla n_{\downarrow}\right|$, or $|\nabla n| \rightarrow 0$, respectively, and those factors can be handled elsewhere in the code, and $\frac{\partial f_{x c}\left(n_{\uparrow}, n_{\downarrow},\left|\nabla n_{\uparrow}\right|,\left|\nabla n_{\downarrow},\right|, \nabla n \mid\right)}{\partial\left|\nabla n_{\uparrow}\right|}, \frac{\partial f_{x c}\left(n_{\uparrow}, n_{\downarrow},\left|\nabla n_{\uparrow}\right|,\left|\nabla n_{\downarrow}\right|,|\nabla n|\right)}{\partial\left|\nabla n_{\downarrow}\right|}$, and $\frac{\partial f_{x c}\left(n_{\uparrow}, n_{\downarrow},\left|\nabla n_{\uparrow}\right|,\left|\nabla n_{\downarrow}\right|,|\nabla n|\right)}{\partial \nabla n \mid}$ can be given out from the functional subroutine. However, there is no consensus in codes about this matter and care needs to be taken in order to make sure that the right quantities are output.

The AM05 subroutine provided at the AM05 website ${ }^{19}$ gives output for the White and Bird scheme: $\underline{\partial f_{x c}\left(n_{\uparrow}, n_{\downarrow},\left|\nabla n_{\uparrow},,\right| \nabla n_{\Downarrow}|,| \nabla n \mid\right)}, \underline{\partial f_{x c}\left(n_{\uparrow}, n_{\downarrow},\left|\nabla n_{\uparrow}\right|,\left|\nabla n_{\downarrow},\right|,|\nabla n|\right)}$, $\frac{\partial f_{x c}\left(n_{\uparrow}, n_{\downarrow},\left|\nabla n_{\uparrow}\right|,\left|\nabla n_{\downarrow}\right|,|\nabla n|\right)}{\partial\left|\nabla n_{\uparrow}\right|}, \quad \frac{\partial f_{x c}\left(n_{\uparrow}, n_{\downarrow},\left|\nabla n_{\uparrow}\right|,\left|\nabla n_{\downarrow}\right|,|\nabla n|\right)}{\partial\left|\nabla n_{\downarrow}\right|}, \quad{ }^{\partial n_{\downarrow}} \quad$ and $\frac{\partial f_{x c}\left(n_{\uparrow}, n_{\downarrow},,\left|\nabla n_{\uparrow},\right| \nabla n_{\downarrow}|,| \nabla n \mid\right)}{\partial|\nabla n|}$. If instead the Pople, Gill, and Johnson output is needed it can be obtained from these quantities by using that

$$
\begin{aligned}
2 & \frac{\partial f_{x c}\left(n_{\uparrow}, n_{\downarrow},\left|\nabla n_{\uparrow}\right|^{2},\left|\nabla n_{\downarrow}\right|^{2}, \nabla n_{\uparrow} \cdot \nabla n_{\downarrow}\right)}{\partial\left|\nabla n_{\uparrow}\right|^{2}} \\
= & \frac{1}{\left|\nabla n_{\uparrow}\right|} \frac{\partial f_{x c}\left(n_{\uparrow}, n_{\downarrow},\left|\nabla n_{\uparrow}\right|,\left|\nabla n_{\downarrow}\right|,|\nabla n|\right)}{\partial\left|\nabla n_{\uparrow}\right|} \\
& +\frac{1}{|\nabla n|} \frac{\partial f_{x c}\left(n_{\uparrow}, n_{\downarrow},\left|\nabla n_{\uparrow}\right|,\left|\nabla n_{\downarrow}\right|,|\nabla n|\right)}{\partial|\nabla n|},
\end{aligned}
$$




$$
\begin{array}{r}
2 \frac{\partial f_{x c}\left(n_{\uparrow}, n_{\downarrow},\left|\nabla n_{\uparrow}\right|^{2},\left|\nabla n_{\downarrow}\right|^{2}, \nabla n_{\uparrow} \cdot \nabla n_{\downarrow}\right)}{\partial\left|\nabla n_{\downarrow}\right|^{2}} \\
=\frac{1}{\left|\nabla n_{\downarrow}\right|} \frac{\partial f_{x c}\left(n_{\uparrow}, n_{\downarrow},\left|\nabla n_{\uparrow}\right|,\left|\nabla n_{\downarrow}\right|,|\nabla n|\right)}{\partial\left|\nabla n_{\downarrow}\right|} \\
\quad+\frac{1}{|\nabla n|} \frac{\partial f_{x c}\left(n_{\uparrow}, n_{\downarrow},\left|\nabla n_{\uparrow}\right|,\left|\nabla n_{\downarrow}\right|,|\nabla n|\right)}{\partial|\nabla n|}, \\
\frac{\partial f_{x c}\left(n_{\uparrow}, n_{\downarrow},\left|\nabla n_{\uparrow}\right|^{2},\left|\nabla n_{\downarrow}\right|^{2}, \nabla n_{\uparrow} \cdot \nabla n_{\downarrow}\right)}{\partial \nabla n_{\uparrow} \cdot \nabla n_{\downarrow}} \\
=\frac{1}{|\nabla n|} \frac{\partial f_{x c}\left(n_{\uparrow}, n_{\downarrow},\left|\nabla n_{\uparrow}\right|,\left|\nabla n_{\downarrow}\right|,|\nabla n|\right)}{\partial|\nabla n|} .
\end{array}
$$

This same output can also be used in calculations of the GGA correction for the stress tensor in Eq. (B2). The terms on the second line of Eq. (B2) constitutes the GGA correction and it can be shown that

$$
\begin{aligned}
\sum_{\xi=\uparrow, \downarrow} & \frac{\partial f_{x c}\left(n_{\uparrow}, n_{\downarrow}, \nabla n_{\uparrow}, \nabla n_{\downarrow}\right)}{\partial\left(\frac{\partial n_{\xi}}{\partial r_{\beta}}\right)} \frac{\partial n_{\xi}}{\partial r_{\alpha}} \\
= & \sum_{\xi=\uparrow, \downarrow} 2 \frac{\partial f_{x c}\left(n_{\uparrow}, n_{\downarrow},\left|\nabla n_{\uparrow}\right|^{2},\left|\nabla n_{\downarrow}\right|^{2}, \nabla n_{\uparrow} \cdot \nabla n_{\downarrow}\right)}{\partial\left|\nabla n_{\xi}\right|^{2}} \frac{\partial n_{\xi}}{\partial r_{\beta}} \frac{\partial n_{\xi}}{\partial r_{\alpha}} \\
& +\frac{\partial f_{x c}\left(n_{\uparrow}, n_{\downarrow},\left|\nabla n_{\uparrow}\right|^{2},\left|\nabla n_{\downarrow}\right|^{2}, \nabla n_{\uparrow} \cdot \nabla n_{\downarrow}\right)}{\partial \nabla n_{\uparrow} \cdot \nabla n_{\downarrow}} \\
& \times\left(\frac{\partial n_{\uparrow}}{\partial r_{\beta}} \frac{\partial n_{\downarrow}}{\partial r_{\alpha}}+\frac{\partial n_{\downarrow}}{\partial r_{\beta}} \frac{\partial n_{\uparrow}}{\partial r_{\alpha}}\right) \\
= & \sum_{\eta=\uparrow, \downarrow, 0} \frac{\partial f_{x c}\left(n_{\uparrow}, n_{\downarrow},\left|\nabla n_{\uparrow}\right|,\left|\nabla n_{\downarrow}\right|,|\nabla n|\right)}{\partial\left|\nabla n_{\eta}\right|} \frac{1}{\left|\nabla n_{\eta}\right|} \frac{\partial n_{\eta}}{\partial r_{\beta}} \frac{\partial n_{\eta}}{\partial r_{\alpha}},
\end{aligned}
$$

where $n_{0}=n=n_{\uparrow}+n_{\downarrow}$, and we again have given the Pople, Gill, and Johnson [Eq. (B11)], and the White and Bird [Eq. (B12)] relevant formulas. We recognize the same derivatives of $f_{x c}$ as appear in Eqs. (B6) and (B7).

In the traditional scheme the second term in Eq. (B3) is further expanded using Eq. (B7):

$$
\begin{aligned}
\nabla \cdot & \frac{\partial f_{x c}\left(n_{\uparrow}, n_{\downarrow}, \nabla n_{\uparrow}, \nabla n_{\downarrow}\right)}{\partial \nabla n_{\nu}} \\
= & \left(\nabla \cdot \frac{\nabla n_{\nu}}{\left|\nabla n_{\nu}\right|}\right) \frac{\partial f_{x c}\left(n_{\uparrow}, n_{\downarrow},\left|\nabla n_{\uparrow}\right|,\left|\nabla n_{\downarrow}\right|,|\nabla n|\right)}{\partial\left|\nabla n_{\nu}\right|} \\
& +\left(\nabla \cdot \frac{\nabla n}{|\nabla n|}\right) \frac{\partial f_{x c}\left(n_{\uparrow}, n_{\downarrow},\left|\nabla n_{\uparrow}\right|,\left|\nabla n_{\downarrow}\right|,|\nabla n|\right)}{\partial|\nabla n|} \\
& +\frac{\nabla n_{\nu}}{\left|\nabla n_{\nu}\right|} \cdot\left(\nabla \frac{\partial f_{x c}\left(n_{\uparrow}, n_{\downarrow},\left|\nabla n_{\uparrow}\right|,\left|\nabla n_{\downarrow}\right|,|\nabla n|\right)}{\partial\left|\nabla n_{\nu}\right|}\right) \\
& +\frac{\nabla n}{|\nabla n|} \cdot\left(\nabla \frac{\partial f_{x c}\left(n_{\uparrow}, n_{\downarrow},\left|\nabla n_{\uparrow}\right|,\left|\nabla n_{\downarrow}\right|,|\nabla n|\right)}{\partial|\nabla n|}\right) .
\end{aligned}
$$

It is straightforward to show that

$$
\nabla \cdot \frac{\nabla n_{\eta}}{\left|\nabla n_{\eta}\right|}=\frac{\nabla^{2} n_{\eta}}{\left|\nabla n_{\eta}\right|}-\frac{\nabla n_{\eta} \cdot \nabla\left|\nabla n_{\eta}\right|}{\left|\nabla n_{\eta}\right|^{2}} \text { for } \eta=\uparrow, \downarrow, 0,
$$

and the two last terms in Eq. (B13) can also be further expanded by using that

$$
\begin{aligned}
\nabla g\left(n_{\uparrow}, n_{\downarrow},\left|\nabla n_{\uparrow}\right|,\left|\nabla n_{\downarrow}\right|,|\nabla n|\right) \\
=\nabla n_{\uparrow} \frac{\partial g}{\partial n_{\uparrow}}+\nabla n_{\downarrow} \frac{\partial g}{\partial n_{\downarrow}}+\nabla\left|\nabla n_{\uparrow}\right| \frac{\partial g}{\partial\left|\nabla n_{\uparrow}\right|}+\nabla\left|\nabla n_{\downarrow}\right| \frac{\partial g}{\partial\left|\nabla n_{\downarrow}\right|} \\
\quad+\nabla|\nabla n| \frac{\partial g}{\partial|\nabla n|} .
\end{aligned}
$$

However, the final general formula would be very cumbersome and we thus now leave generality and instead make use of specific dependencies existing in GGA-type functionals, focusing on the AM05 functional.

The exchange terms in AM05 are only dependent on either spin-up or spin-down densities. This is also the case for GGA exchange in general. The dependency of the correlation part of the spin-dependent AM05 functional is $f_{c}$ $=f_{c}\left(n_{\uparrow}, n_{\downarrow},\left|\nabla n_{\uparrow}\right|\right)+f_{c}\left(n_{\uparrow}, n_{\downarrow},\left|\nabla n_{\downarrow}\right|\right)$ while, for example, PBE has another dependency: $f_{c}=f_{c}\left(n_{\uparrow}, n_{\downarrow},|\nabla n|\right)$. It follows from Eqs. (B13)-(B15) that all these forms give, for $\nu=\uparrow$ and $\downarrow$,

$$
\begin{aligned}
\nabla \cdot \frac{\partial f\left(n_{\uparrow}, n_{\downarrow},\left|\nabla n_{\eta}\right|\right)}{\partial \nabla n_{\nu}}= & \left(\frac{\nabla^{2} n_{\eta}}{\left|\nabla n_{\eta}\right|}-\frac{\nabla n_{\eta} \cdot \nabla\left|\nabla n_{\eta}\right|}{\left|\nabla n_{\eta}\right|^{2}}\right) \frac{\partial f\left(n_{\uparrow}, n_{\downarrow},\left|\nabla n_{\eta}\right|\right)}{\partial\left|\nabla n_{\eta}\right|}+\frac{\nabla n_{\eta}}{\left|\nabla n_{\eta}\right|} \cdot\left(\sum_{\xi=\uparrow, \downarrow} \nabla n_{\xi} \frac{\partial^{2} f\left(n_{\uparrow}, n_{\downarrow},\left|\nabla n_{\eta}\right|\right)}{\partial n_{\xi} \partial\left|\nabla n_{\eta}\right|}+\nabla\left|\nabla n_{\eta}\right| \frac{\partial^{2} f\left(n_{\uparrow}, n_{\downarrow},\left|\nabla n_{\eta}\right|\right)}{\partial\left|\nabla n_{\eta}\right|^{2}}\right) \\
= & \nabla^{2} n_{\eta}\left(\frac{1}{\left|\nabla n_{\eta}\right|} \frac{\partial f\left(n_{\uparrow}, n_{\downarrow},\left|\nabla n_{\eta}\right|\right)}{\partial\left|\nabla n_{\eta}\right|}\right)+\sum_{\xi=\uparrow, \downarrow} \frac{\nabla n_{\eta} \cdot \nabla n_{\xi}}{\left|\nabla n_{\eta}\right|} \frac{\partial^{2} f\left(n_{\uparrow}, n_{\downarrow},\left|\nabla n_{\eta}\right|\right)}{\partial n_{\xi} \partial\left|\nabla n_{\eta}\right|} \\
& +\nabla n_{\eta} \cdot \nabla\left|\nabla n_{\eta}\right| \frac{\partial}{\partial\left|\nabla n_{\eta}\right|}\left(\frac{1}{\left|\nabla n_{\eta}\right|} \frac{\partial f\left(n_{\uparrow}, n_{\downarrow},\left|\nabla n_{\eta}\right|\right)}{\partial\left|\nabla n_{\eta}\right|}\right),
\end{aligned}
$$


when $\eta=\nu$ or 0 , and 0 if $\eta$ is the opposite spin to $\nu$. We have here suppressed the index denoting exchange and correlation. For the exchange, one of the terms in the sum over spin up and spin down will vanish since the exchange part of the functional only depends on one spin and thus the cross-spin derivative will be zero. The density gradient $\nabla n_{\eta} \cdot \nabla n_{\xi}$ is a function only of $\left|\nabla n_{\eta}\right|, \eta=\uparrow, \downarrow, 0$, which can be made explicit by using that $\nabla n_{\uparrow} \cdot \nabla n_{\downarrow}=\left(|\nabla n|^{2}-\left|\nabla n_{\uparrow}\right|^{2}-\left|\nabla n_{\downarrow}\right|^{2}\right) / 2$.

We notice that derivatives of the functional and derivatives of the densities no longer are intermingled but separated and if the density derivatives $\nabla^{2} n_{\eta}$ and $\nabla n_{\eta} \cdot \nabla\left|\nabla n_{\eta}\right|$ are handled into a functional routine in addition to the already required $\eta_{\uparrow}, \eta_{\downarrow}$, and $\left|\nabla n_{\eta}\right|$, the two exchange-correlation potentials can be assembled. Note that even if the quantity $\nabla n_{\eta} \cdot \nabla\left|\nabla n_{\eta}\right|$ looks complicated it is easily shown that

$$
\nabla n_{\eta} \cdot \nabla\left|\nabla n_{\eta}\right|=\frac{1}{\left|\nabla n_{\eta}\right|} \sum_{i=1}^{3} \sum_{j=1}^{3} \frac{\partial n_{\eta}}{\partial r_{i}} \frac{\partial n_{\eta}}{\partial r_{j}} \frac{\partial^{2} n_{\eta}}{\partial r_{i} \partial r_{j}}
$$

where $r_{1}=r_{x}, r_{2}=r_{y}$, and $r_{3}=r_{z}$.

In the following more detailed manipulations, we will only treat AM05, leaving, for example, the derivation of the PBE correlation potentials as an exercise. For ease we divide up the AM05 exchange-correlation energy density in Eq. (14) in separate exchange and correlation parts:

$$
\begin{aligned}
f_{x}\left(n_{\uparrow}, n_{\downarrow},\left|\nabla n_{\uparrow}\right|,\left|\nabla n_{\downarrow}\right|\right) & \\
= & \frac{1}{2}\left[f_{x}^{\mathrm{LDA}}\left(2 n_{\uparrow}\right) H_{x}\left(s_{\uparrow}\right)+f_{x}^{\mathrm{LDA}}\left(2 n_{\downarrow}\right) H_{x}\left(s_{\downarrow}\right)\right],
\end{aligned}
$$

where $f_{x}^{\mathrm{LDA}}(n)=n \epsilon_{x}^{\mathrm{LDA}}(n)$ is the LDA exchange energy density, and

$$
\begin{aligned}
& f_{c}\left(n_{\uparrow}, n_{\downarrow},\left|\nabla n_{\uparrow}\right|,\left|\nabla n_{\downarrow}\right|\right) \\
& \quad=f_{c}^{\mathrm{LDA}}\left(n_{\uparrow}, n_{\downarrow}\right)\left(\frac{n_{\uparrow}}{\left(n_{\uparrow}+n_{\downarrow}\right)} H_{c}\left(s_{\uparrow}\right)+\frac{n_{\downarrow}}{\left(n_{\uparrow}+n_{\downarrow}\right)} H_{c}\left(s_{\downarrow}\right)\right),
\end{aligned}
$$

where $f_{c}^{\mathrm{LDA}}\left(n_{\uparrow}, n_{\downarrow}\right)=\left(n_{\uparrow}+n_{\downarrow}\right) \epsilon_{c}^{\mathrm{LDA}}\left(n_{\uparrow}, n_{\downarrow}\right)$ is the LDA correlation energy density.

Using that

$$
\begin{aligned}
& \frac{\partial s(n, \nabla n)}{\partial n}=-\frac{4}{3} \frac{s}{n}, \\
& \frac{\partial s(n, \nabla n)}{\partial|\nabla n|}=\frac{1}{2 k_{F} n},
\end{aligned}
$$

we obtain from Eq. (B18)

$$
\begin{aligned}
& \frac{\partial f_{x}\left(n_{\uparrow}, n_{\downarrow},\left|\nabla n_{\uparrow}\right|,\left|\nabla n_{\downarrow}\right|\right)}{\partial n_{\nu}} \\
& =\frac{\partial f_{x}^{\mathrm{LDA}}\left(2 n_{\nu}\right)}{\partial\left(2 n_{\nu}\right)} H_{x}\left(s_{\nu}\right)-f_{x}^{\mathrm{LDA}}\left(2 n_{\nu}\right) \frac{4}{3} \frac{s_{\nu}}{2 n_{\nu}} \frac{\partial H_{x}\left(s_{\nu}\right)}{\partial s_{\nu}} \\
& =v_{x}^{\mathrm{LDA}}\left(2 n_{\nu}\right) H_{x}\left(s_{\nu}\right)-\frac{4}{3} \epsilon_{x}^{\mathrm{LDA}}\left(2 n_{\nu}\right) s_{\nu} \frac{\partial H_{x}\left(s_{\nu}\right)}{\partial s_{\nu}},
\end{aligned}
$$

and

$$
\begin{aligned}
\frac{\partial f_{x}\left(n_{\uparrow}, n_{\downarrow},\left|\nabla n_{\uparrow}\right|,\left|\nabla n_{\downarrow}\right|\right)}{\partial\left|\nabla n_{\nu}\right|} & =f_{x}^{\mathrm{LDA}}\left(2 n_{\nu}\right) \frac{1}{2 k_{F, \nu}\left(2 n_{\nu}\right)} \frac{\partial H_{x}\left(s_{\nu}\right)}{\partial s_{\nu}} \\
& =\frac{\epsilon_{x}^{\mathrm{LDA}}\left(2 n_{\nu}\right)}{2 k_{F, \nu}} \frac{\partial H_{x}\left(s_{\nu}\right)}{\partial s_{\nu}},
\end{aligned}
$$

where $k_{F, \nu}=k_{F}\left(2 n_{\nu}\right), v_{x}^{\mathrm{LDA}}\left(2 n_{\nu}\right)$ is the LDA spin- $\nu$ exchange potential, and $\epsilon_{x}^{\mathrm{LDA}}\left(2 n_{\nu}\right)$ is the LDA spin- $\nu$ exchange energy per particle.

Using Eq. (B19) we obtain

$$
\begin{aligned}
\frac{\partial f_{c}\left(n_{\uparrow}, n_{\downarrow},\left|\nabla n_{\uparrow}\right|,\left|\nabla n_{\downarrow}\right|\right)}{\partial n_{\nu_{a}}}= & \frac{\partial f_{c}^{\mathrm{LDA}}\left(n_{\uparrow}, n_{\downarrow}\right)}{\partial n_{\nu_{a}}}\left[\frac{n_{\uparrow}}{\left(n_{\uparrow}+n_{\downarrow}\right)} H_{c}\left(s_{\uparrow}\right)+\frac{n_{\downarrow}}{\left(n_{\uparrow}+n_{\downarrow}\right)} H_{c}\left(s_{\downarrow}\right)\right]+f_{c}^{\mathrm{LDA}}\left(n_{\uparrow}, n_{\downarrow}\right) \\
& \times\left\{\frac{\partial\left[\frac{n_{\nu_{b}}}{\left(n_{\uparrow}+n_{\downarrow}\right)}\right]}{\partial n_{\nu_{a}}} H_{c}\left(s_{\nu_{b}}\right)\right\}+f_{c}^{\mathrm{LDA}}\left(n_{\uparrow}, n_{\downarrow}\right) \frac{2 n_{\nu_{a}}}{\left(n_{\uparrow}+n_{\downarrow}\right)} \frac{\partial H_{c}\left(s_{\nu_{a}}\right)}{\partial n_{\nu_{a}}} H_{c}\left(s_{\nu_{a}}\right)+\frac{\left.n_{\nu_{a}}\right)}{n_{\nu_{b}}}\left[H_{c}\left(s_{\nu_{a}}\right)-H_{c}\left(s_{\nu_{b}}\right)\right] \\
= & v_{c, \nu_{a}}^{\mathrm{LDA}}\left(n_{\uparrow}, n_{\downarrow}\right) \frac{1}{\left(n_{\uparrow}+n_{\downarrow}\right)}\left[n_{\uparrow} H_{c}\left(s_{\uparrow}\right)+n_{\downarrow} H_{c}\left(s_{\downarrow}\right)\right]+\epsilon_{c}^{\mathrm{LDA}}\left(n_{\uparrow}, n_{\downarrow}\right) \frac{\left.n_{\uparrow}+n_{\downarrow}\right)}{\left(n_{\downarrow}\right.} \\
& -\frac{4}{3} \epsilon_{c}^{\mathrm{LDA}}\left(n_{\uparrow}, n_{\downarrow}\right) s_{\nu_{a}} \frac{\partial H_{c}\left(s_{\nu_{a}}\right)}{\partial s_{\nu_{a}}}
\end{aligned}
$$

where $\nu_{b}$ is the opposite spin to $\nu_{a}$, and

$$
\frac{\partial f_{c}\left(n_{\uparrow}, n_{\downarrow},\left|\nabla n_{\uparrow}\right|,\left|\nabla n_{\downarrow}\right|\right)}{\partial\left|\nabla n_{\nu}\right|}=f_{c}^{\mathrm{LDA}}\left(n_{\uparrow}, n_{\downarrow}\right) \frac{2 n_{\nu}}{\left(n_{\uparrow}+n_{\downarrow}\right)} \frac{\partial H_{c}\left(s_{\nu}\right)}{\partial\left|\nabla\left(2 n_{\nu}\right)\right|}=\frac{\epsilon_{c}^{\mathrm{LDA}}\left(n_{\uparrow}, n_{\downarrow}\right)}{2 k_{F, \nu}} \frac{\partial H_{c}\left(s_{\nu}\right)}{\partial s_{\nu}},
$$


where $v_{c, \nu}^{\mathrm{LDA}}\left(n_{\uparrow}, n_{\downarrow}\right)$ is the LDA spin- $\nu$ correlation potential and $\epsilon_{c}^{\mathrm{LDA}}\left(n_{\uparrow}, n_{\downarrow}\right)$ is the LDA correlation energy per particle.

The quantities in Eqs. (B22)-(B25) gives the input needed; $\frac{\partial f_{x c}}{\partial n_{v_{a}}}=\frac{\partial f_{x}}{\partial n_{v_{a}}}+\frac{\partial f_{c}}{\partial n_{v_{a}}}$, giving Eq. (25), and $\frac{\partial f_{x c}}{\partial\left|\nabla n_{v_{a}}\right|}=\frac{\partial f_{x}}{\partial\left|\nabla n_{v_{a}}\right|}$ $+\frac{\partial f_{c}}{\partial\left|\nabla n_{v_{a}}\right|}$, giving Eq. (26), in the White and Bird and the Pople, Gill, and Johnson schemes for constructing the exchangecorrelation spin- $\nu_{a}$ potential outside of the functional subroutine [see, however, the note below Eqs. (B6) and (B7)].

For the traditional scheme, starting with exchange, we obtain from Eq. (B16), Eqs. (B22) and (B23), and the definitions in Eqs. (10) and (27):

$$
\begin{aligned}
\nabla \cdot & \frac{\partial f_{x}\left(n_{\uparrow}, n_{\downarrow}, \nabla n_{\uparrow}, \nabla n_{\downarrow}\right)}{\partial \nabla n_{\nu}} \\
= & t_{\nu} \epsilon_{x}^{\mathrm{LDA}}\left(2 n_{\nu}\right) \frac{1}{s_{\nu}} \frac{\partial H_{x}\left(s_{\nu}\right)}{\partial s_{\nu}}+v_{x}^{\mathrm{LDA}}\left(2 n_{\nu}\right) s_{\nu} \frac{\partial H_{x}\left(s_{\nu}\right)}{\partial s_{\nu}} \\
& -\frac{4}{3} \epsilon_{x}^{\mathrm{LDA}}\left(2 n_{\nu}\right) s_{\nu} \frac{\partial}{\partial s_{\nu}}\left(s_{\nu} \frac{\partial H_{x}\left(s_{\nu}\right)}{\partial s_{\nu}}\right) \\
& +u_{\nu} \epsilon_{x}^{\mathrm{LDA}}\left(2 n_{\nu}\right) \frac{\partial}{\partial s_{\nu}}\left(\frac{1}{s_{\nu}} \frac{\partial H_{x}\left(s_{\nu}\right)}{\partial s_{\nu}}\right) .
\end{aligned}
$$

In order to reduce the number of different derivatives of $H(s)$, we use that

$$
s \frac{\partial}{\partial s}\left(s \frac{\partial H(s)}{\partial s}\right)-s \frac{\partial H(s)}{\partial s}=s^{3} \frac{\partial}{\partial s}\left(\frac{1}{s} \frac{\partial H(s)}{\partial s}\right)+s \frac{\partial H(s)}{\partial s},
$$

and, from Eqs. (B3), (B22), and (B26), we arrive at the final form of the exchange part of the spin- $\nu$ exchange-correlation potential:

$$
\begin{aligned}
V_{x, \nu}= & v_{x}^{\mathrm{LDA}}\left(2 n_{\nu}\right)\left[H_{x}\left(s_{\nu}\right)-s_{\nu} \frac{\partial H_{x}\left(s_{\nu}\right)}{\partial s_{\nu}}\right]+\epsilon_{x}^{\mathrm{LDA}}\left(2 n_{\nu}\right) \\
& \times\left\{\left(\frac{4}{3} s_{\nu}^{2}-t_{\nu}\right) \frac{1}{s_{\nu}} \frac{\partial H_{x}\left(s_{\nu}\right)}{\partial s_{\nu}}\right. \\
& \left.+\left(\frac{4}{3} s_{\nu}^{3}-u_{\nu}\right) \frac{\partial}{\partial s_{\nu}}\left[\frac{1}{s_{\nu}} \frac{\partial H_{x}\left(s_{\nu}\right)}{\partial s_{\nu}}\right]\right\} .
\end{aligned}
$$

For correlation, by a similar procedure starting from Eqs. (B24) and (B25) and again using Eq. (B16), we get

$$
\begin{aligned}
& \nabla \cdot \frac{\partial f_{c}\left(n_{\uparrow}, n_{\downarrow}, \nabla n_{\uparrow}, \nabla n_{\downarrow}\right)}{\partial \nabla n_{\nu_{a}}}=t_{\nu_{a}} \epsilon_{c}^{\mathrm{LDA}}\left(n_{\uparrow}, n_{\downarrow}\right) \frac{1}{s_{\nu_{a}}} \frac{\partial H_{c}\left(s_{\nu_{a}}\right)}{\partial s_{\nu_{a}}}+\left(v_{c, \nu_{a}}^{\mathrm{LDA}}\left(n_{\uparrow}, n_{\downarrow}\right)+\frac{\nabla n_{\uparrow} \cdot \nabla n_{\downarrow}}{\left|\nabla n_{\nu_{a}}\right|^{2}} v_{c, \nu_{b}}^{\mathrm{LDA}}\left(n_{\uparrow}, n_{\downarrow}\right)\right) \frac{n_{\nu_{a}}}{n_{\uparrow}+n_{\downarrow}} s_{\nu_{a}} \frac{\partial H_{c}\left(s_{\nu_{a}}\right)}{\partial s_{\nu_{a}}} \\
& +\left(n_{\nu_{b}}-\frac{\nabla n_{\uparrow} \cdot \nabla n_{\downarrow}}{\left|\nabla n_{\nu_{a}}\right|^{2}} n_{\nu_{a}}\right) \frac{\epsilon_{c}^{\mathrm{LDA}}\left(n_{\uparrow}, n_{\downarrow}\right)}{n_{\uparrow}+n_{\downarrow}} s_{v_{a}} \frac{\partial H_{c}\left(s_{\nu_{a}}\right)}{\partial s_{\nu_{a}}}-\frac{4}{3} \epsilon_{c}^{\mathrm{LDA}}\left(n_{\uparrow}, n_{\downarrow}\right) s_{v_{a}} \frac{\partial}{\partial s_{\nu_{a}}}\left(s_{\nu_{a}} \frac{\partial H_{c}\left(s_{\nu_{a}}\right)}{\partial s_{\nu_{a}}}\right) \\
& +u_{\nu_{a}} \epsilon_{c}^{\mathrm{LDA}}\left(n_{\uparrow}, n_{\downarrow}\right) \frac{\partial}{\partial s_{\nu_{a}}}\left(\frac{1}{s_{\nu_{a}}} \frac{\partial H_{c}\left(s_{\nu_{a}}\right)}{\partial s_{v_{a}}}\right) .
\end{aligned}
$$

Using Eqs. (B3), (B24), (B27), and (B9), we arrive at

$$
\begin{aligned}
& V_{c, \nu_{a}}=v_{c, v_{a}}^{\mathrm{LDA}}\left(n_{\uparrow}, n_{\downarrow}\right)\left[H_{c}\left(s_{v_{a}}\right)-s_{\nu_{a}} \frac{\partial H_{c}\left(s_{\nu_{a}}\right)}{\partial s_{\nu_{a}}}\right]+\epsilon_{c}^{\mathrm{LDA}}\left(n_{\uparrow}, n_{\downarrow}\right)\left\{\left(\frac{4}{3} s_{v_{a}}^{2}-t_{v_{a}}\right) \frac{1}{s_{v_{a}}} \frac{\partial H_{c}\left(s_{v_{a}}\right)}{\partial s_{v_{a}}}+\left(\frac{4}{3} s_{v_{a}}^{3}-u_{v_{a}}\right) \frac{\partial}{\partial s_{\nu_{a}}}\left[\frac{1}{s_{\nu_{a}}} \frac{\partial H_{c}\left(s_{\nu_{a}}\right)}{\partial s_{v_{a}}}\right]\right\} \\
& +\left[\epsilon_{c}^{\mathrm{LDA}}\left(n_{\uparrow}, n_{\downarrow}\right)-v_{c, \nu_{a}}^{\mathrm{LDA}}\left(n_{\uparrow}, n_{\downarrow}\right)\right] \frac{n_{\nu_{b}}}{n_{\uparrow}+n_{\downarrow}}\left[H_{c}\left(s_{\nu_{a}}\right)-H_{c}\left(s_{\nu_{b}}\right)-s_{\nu_{a}} \frac{\partial H_{c}\left(s_{\nu_{a}}\right)}{\partial s_{\nu_{a}}}\right] \\
& +\left[\epsilon_{c}^{\mathrm{LDA}}\left(n_{\uparrow}, n_{\downarrow}\right)-v_{c, v_{b}}^{\mathrm{LDA}}\left(n_{\uparrow}, n_{\downarrow}\right)\right] \frac{\nabla n_{\uparrow} \cdot \nabla n_{\downarrow}}{\left|\nabla n_{\nu_{a}}\right|^{2}} \frac{n_{\nu_{a}}}{n_{\uparrow}+n_{\downarrow}} s_{\nu_{a}} \frac{\partial H_{c}\left(s_{v_{a}}\right)}{\partial s_{v_{a}}} .
\end{aligned}
$$

The total spin- $\nu_{a}$ exchange-correlation potential, $V_{x c, \nu_{a}}=V_{x, \nu_{a}}+V_{c, \nu_{a}}$, is given in Eq. (28).

For implementation into codes also the various $s$ derivatives of $H_{x}(s)$ and $H_{c}(s)$ in Eqs. (B22)-(B25), (B28), and (B30) are needed. We will here adhere closely to the notation used in the subroutines provided at the AM05 web page. ${ }^{19}$ We have

$$
\begin{gathered}
\frac{1}{s} \frac{\partial H_{x}(s)}{\partial s}=[1-X(s)] \frac{1}{s} \frac{\partial F_{x}^{\mathrm{LAA}}(s)}{\partial s}+\left[1-F_{x}^{\mathrm{LAA}}(s)\right] \frac{1}{s} \frac{\partial X(s)}{\partial s}, \\
\frac{1}{s} \frac{\partial H_{c}(s)}{\partial s}=[1-\gamma] \frac{1}{s} \frac{\partial X(s)}{\partial s},
\end{gathered}
$$

and 


$$
\begin{gathered}
\frac{1}{s} \frac{\partial}{\partial s}\left(\frac{1}{s} \frac{\partial H_{x}(s)}{\partial s}\right)=[1-X(s)] \frac{1}{s} \frac{\partial}{\partial s}\left(\frac{1}{s} \frac{\partial F_{x}^{\mathrm{LAA}}(s)}{\partial s}\right)+\left[1-F_{x}^{\mathrm{LAA}}(s)\right] \frac{1}{s} \frac{\partial}{\partial s}\left(\frac{1}{s} \frac{\partial X(s)}{\partial s}\right)-2\left(\frac{1}{s} \frac{\partial X(s)}{\partial s}\right)\left(\frac{1}{s} \frac{\partial F_{x}^{\mathrm{LAA}}(s)}{\partial s}\right), \\
\frac{1}{s} \frac{\partial}{\partial s}\left(\frac{1}{s} \frac{\partial H_{c}(s)}{\partial s}\right)=[1-\gamma] \frac{1}{s} \frac{\partial}{\partial s}\left(\frac{1}{s} \frac{\partial X(s)}{\partial s}\right),
\end{gathered}
$$

where

$$
\begin{gathered}
\frac{1}{s} \frac{\partial X(s)}{\partial s}=-2 \alpha[X(s)]^{2}, \\
\frac{1}{s} \frac{\partial}{\partial s}\left(\frac{1}{s} \frac{\partial X(s)}{\partial s}\right)=8 \alpha^{2}[X(s)]^{3},
\end{gathered}
$$

and $X(s)$ is given in Eq. (4). The derivatives of $F_{x}^{\mathrm{LAA}}(s)$ are more elaborate. Using the definitions in Eqs. (17) and (18) we obtain

$$
\frac{1}{s} \frac{\partial F_{x}^{\mathrm{LAA}}(s)}{\partial s}=\frac{1}{s} \frac{c 2 s D(s)-\left(c s^{2}+1\right) \frac{d D(s)}{d s}}{[D(s)]^{2}}=\frac{U(s)}{[D(s)]^{2}}=\frac{c}{[D(s)]^{2}}\left[2-\left(1+c s^{2}\right) \frac{d A(s)}{d s}-\left(1-c s^{2}\right) \frac{A(s)}{s}\right],
$$

where $A(s)$ is given in Eq. (19) and $d A(s) / d s$ is given below, in Eq. (B41). Lastly, using the definition of $U(s)$ on the first line of Eq. (B37), we find

$$
\begin{aligned}
\frac{1}{s} \frac{\partial}{\partial s}\left(\frac{1}{s} \frac{\partial F_{x}^{\mathrm{LAA}}(s)}{\partial s}\right) & =\frac{1}{s} \frac{\frac{d U(s)}{d s} D(s)-2 U(s) \frac{d D(s)}{d s}}{[D(s)]^{3}} \\
& =\frac{-4 c s^{2} D(s)\left(\frac{1}{s} \frac{d D(s)}{d s}\right)+\left(c s^{2}+1\right)\left\{2 s^{2}\left(\frac{1}{s} \frac{d D(s)}{d s}\right)^{2}-D(s)\left[s \frac{d}{d s}\left(\frac{1}{s} \frac{d D(s)}{d s}\right)\right]\right\}}{s^{2}[D(s)]^{3}},
\end{aligned}
$$

where $D(s)$ is the denominator of $F_{x}^{\mathrm{LAA}}(s)$ given in Eq. (18), and

$$
\begin{gathered}
\frac{1}{s} \frac{d D(s)}{d s}=c\left(\frac{d A(s)}{d s}+\frac{A(s)}{s}\right) \\
s \frac{d}{d s}\left(\frac{1}{s} \frac{d D(s)}{d s}\right)=c\left[s \frac{d^{2} A(s)}{d s^{2}}+\left(\frac{d A(s)}{d s}-\frac{A(s)}{s}\right)\right] .
\end{gathered}
$$

The derivatives of $A(s)$ can be worked out from the definition in Eq. (19):

$$
\frac{d A(s)}{d s}=\frac{d Z(s)}{d s}\left\{1+\frac{3}{2}[k Z(s)]^{2}\right\}\left\{1+[k Z(s)]^{2}\right\}^{-3 / 4},
$$

and

$$
\begin{aligned}
s \frac{d^{2} A(s)}{d s^{2}}= & s \frac{d^{2} Z(s)}{d s^{2}}\left\{1+\frac{3}{2}[k Z(s)]^{2}\right\}\left\{1+[k Z(s)]^{2}\right\}^{-3 / 4} \\
& +\left(\frac{d Z(s)}{d s}\right)^{2} \frac{s}{Z(s)}\left\{\frac{3}{2}[k Z(s)]^{2}+\frac{3}{4}[k Z(s)]^{4}\right\} \\
& \times\left\{1+[k Z(s)]^{2}\right\}^{-7 / 4} .
\end{aligned}
$$

Finally, the $s$ derivatives of $Z(s)$ can be derived from its definition in Eq. (21) by using that

$$
\frac{d W(x)}{d x}=\frac{1}{x} \frac{W(x)}{1+W(x)}
$$

which together with the definition of $\chi(s)$ in Eq. (22) gives

$$
\frac{d W(\chi(s))}{d s}=\frac{3}{2} \frac{1}{s} \frac{W(\chi(s))}{1+W(\chi(s))} .
$$

The final ingredients needed for the implementation of the AM05 potentials are thus

$$
\frac{d Z(s)}{d s}=\frac{Z(s)}{s} \frac{1}{1+W(\chi(s))},
$$

and, noting that the $s$ times the second derivative of $Z(s)$ at the first line in Eq. (B42) only will appear together with the first derivative of $Z(s)$ from Eq. (B41), see Eq. (B40), 


$$
\begin{aligned}
s \frac{d^{2} Z(s)}{d s^{2}}+\frac{d Z(s)}{d s} & =\frac{d}{d s}\left[s \frac{d Z(s)}{d s}\right] \\
& =\frac{Z(s)}{s} \frac{1}{[1+W(\chi(s))]^{3}}\left(1-\frac{1}{2} W(\chi(s))\right) .
\end{aligned}
$$

The Fortran routine available at the AM05 web page ${ }^{19}$ consists of several subroutines. The goal has been to provide a stand-alone AM05 core subroutine that does not need to be manipulated but all necessary modifications for adaptation to a specific code should be done in one of several provided template subroutines. The template routines assemble the required input to, and modifies the obtained output from, the AM05 subroutine. The three template routines provided are examples of adaptations to codes using the White and Bird scheme, the Pople, Gill, and Johnson scheme, and the traditional scheme, respectively. Once AM05 has been implemented and tested in this way, subroutines used inside the AM05 subroutine, such as the LDA exchange and correlation routines, can be replaced with routines already in the code. *aematts@sandia.gov

${ }^{1}$ P. Hohenberg and W. Kohn, Phys. Rev. 136, B864 (1964).

${ }^{2}$ W. Kohn and L. J. Sham, Phys. Rev. 140, A1133 (1965).

${ }^{3}$ U. von Barth and L. Hedin, J. Phys. C 5, 1629 (1972).

${ }^{4}$ M. M. Pant and A. K. Rajagopal, Solid State Commun. 10, 1157 (1972).

${ }^{5}$ R. Armiento and A. E. Mattsson, Phys. Rev. B 72, 085108 (2005).

${ }^{6}$ A. E. Mattsson, R. Armiento, J. Paier, G. Kresse, J. M. Wills, and T. R. Mattsson, J. Chem. Phys. 128, 084714 (2008).

${ }^{7}$ M. Ropo, K. Kokko, and L. Vitos, Phys. Rev. B 77, 195445 (2008).

${ }^{8}$ P. Haas, F. Tran, and P. Blaha, Phys. Rev. B 79, 085104 (2009).

${ }^{9}$ J. P. Perdew, K. Burke, and M. Ernzerhof, Phys. Rev. Lett. 77, 3865 (1996).

${ }^{10}$ A. E. Mattsson and W. Kohn, J. Chem. Phys. 115, 3441 (2001).

${ }^{11}$ R. Armiento and A. E. Mattsson, Phys. Rev. B 66, 165117 (2002).

${ }^{12}$ J. P. Perdew and Y. Wang, Phys. Rev. B 45, 13244 (1992).

${ }^{13}$ D. M. Ceperley and B. J. Alder, Phys. Rev. Lett. 45, 566 (1980).

${ }^{14}$ W. Kohn and A. E. Mattsson, Phys. Rev. Lett. 81, 3487 (1998).

${ }^{15}$ N. D. Lang and W. Kohn, Phys. Rev. B 1, 4555 (1970).

${ }^{16}$ Z. Yan, J. P. Perdew, and S. Kurth, Phys. Rev. B 61, 16430 (2000); J. M. Pitarke and A. G. Eguiluz, ibid. 63, 045116 (2001).
${ }^{17}$ L. A. Constantin, J. M. Pitarke, J. F. Dobson, A. Garcia-Lekue, and J. P. Perdew, Phys. Rev. Lett. 100, 036401 (2008).

${ }^{18}$ R. M. Corless, G. H. Gonnet, D. E. G. Hare, D. J. Jeffrey, and D. E. Knuth, Adv. Comput. Math. 5, 329 (1996).

${ }^{19}$ Subroutines are available at the AM05 web site: http:// dft.sandia.gov/functionals/AM05.html.

${ }^{20}$ See EPAPS Document NoE-PRBMDO-79-084911 for a subroutine for calculating the Lambert $\mathrm{W}$ function required in implementations of AM05. This document can be reached via a direct link in the online article's HTML reference section or via the EPAPS homepage (http://www.aip.org/pubservs/epaps.html).

${ }^{21}$ J. A. White and D. M. Bird, Phys. Rev. B 50, 4954 (1994).

${ }^{22}$ J. A. Pople, P. M. W. Gill, and B. G. Johnson, Chem. Phys. Lett. 199, 557 (1992).

${ }^{23}$ G. Kresse and J. Hafner, Phys. Rev. B 47, 558 (1993); 49, 14251 (1994); G. Kresse and J. Furthmüller, ibid. 54, 11169 (1996).

${ }^{24}$ J. Paier, R. Hirschl, M. Marsman, and G. Kresse, J. Chem. Phys. 122, 234102 (2005).

${ }^{25}$ D. E. Jiang and E. A. Carter, Phys. Rev. B 67, 214103 (2003).

${ }^{26}$ A. E. Mattsson, P. A. Schultz, M. P. Desjarlais, T. R. Mattsson, and K. Leung, Modell. Simul. Mater. Sci. Eng. 13, R1 (2005).

${ }^{27}$ H. J. Monkhorst and J. D. Pack, Phys. Rev. B 13, 5188 (1976).

${ }^{28}$ F. D. Murnaghan, Proc. Natl. Acad. Sci. U.S.A. 30, 244 (1944). 